To Appear in the Astrophysical Journal

Preprint typeset using IATEX style emulateapj v. 05/04/06

\title{
AGB CONNECTION AND ULTRAVIOLET LUMINOSITY EXCESS IN ELLIPTICAL GALAXIES
}

\author{
Alberto Buzzoni ${ }^{1}$ And Rosa A. GonzÁlez-Lópezlira ${ }^{2}$ \\ To appear in the Astrophysical Journal
}

\begin{abstract}
Relying on infrared surface brightness fluctuactions to trace AGB properties in a sample of elliptical galaxies in the Virgo and Fornax clusters, we assess the puzzling origin of the "UV-upturn" phenomenon, recently traced down to the presence of a hot horizontal branch (HB) stellar component. We find that the UV-upturn actually signals a profound change in the c-m diagram of stellar populations in elliptical galaxies, involving both the hot stellar component and red-giant evolution. First, we encounter that the strengthening of the UV rising branch is always seen to correspond to a shortening in AGB deployment; this trend can be readily interpreted as an age effect, perhaps mildly modulated by metal abundance.

A comparison between galaxy $\bar{K}$ magnitudes and population synthesis models confirms that, all the way, brightest stars in ellipticals are genuine AGB members, reaching the thermal-pulsing phase, and with the AGB tip exceeding the RGB tip by some 0.5-1.5 mag. The inferred core mass of these stars is found to be $\lesssim 0.57 \mathrm{M}_{\odot}$ among giant ellipticals. Coupled with the recognized severe deficiency of planetary nebulae in these galaxies, this result strongly calls for an even more critical blocking effect due to a lengthy transition time needed by the post-AGB stellar core to become a hard UV emitter and eventually "fire up" the nebula.

The combined study of galaxy $(1550-V)_{o}$ color and integrated $\mathrm{H} \beta$ index points, as an explanation for the UV-upturn phenomenon, to a composite HB with a bimodal temperature distribution, i.e. with both a red clump and an extremely blue component, in a relative proportion of roughly $[\mathrm{N}(\mathrm{RHB}): \mathrm{N}(\mathrm{BHB})] \sim[80: 20]$. As far as metallicity of the BHB stellar population is concerned, we find that $[\mathrm{Fe} / \mathrm{H}]$ values of either $\simeq-0.7 \mathrm{dex}$ or $\gtrsim+0.5$ may provide the optimum ranges to feed the needed low-mass stars $\left(\mathrm{M}_{*} \ll 0.58 \mathrm{M}_{\odot}\right)$, that at some stage begin to join the standard red-clump stars.

Subject headings: stars: AGB and Post-AGB; stars: mass-loss; galaxies: elliptical and lenticular, cD; galaxies: evolution; ultraviolet: galaxies
\end{abstract}

\section{INTRODUCTION AND THEORETICAL FRAMEWORK}

The so-called "UV-upturn" phenomenon (Code \& Welch 1979), i.e., the rising ultraviolet emission shortward of $2000 \AA$, sometimes featuring in the spectral energy distribution (SED) of elliptical galaxies and the bulges of spirals, has been for long a puzzling problem for old galaxy environments dominated by stars of mass comparable to the Sun.

In fact, the implied existence of an important contribution of (long-lived) B stars, hotter than $\sim 30000 \mathrm{~K}$ and providing up to about $2 \%$ of the galaxy bolometric luminosity (Renzini \& Buzzoni 1986), has been alternately identified with different evolutionary stages. Such stages include binaries (Brown et al. 2006), blue stragglers (Bailvn 1995), blue horizontal-branch (HB) stars (Dorman et al. 1995), asymptotic giant branch (AGB) manqué stars (Greggio \& Renzini 1990), and post-AGB nuclei of planetary nebulae $(\mathrm{PNe})$ (Renzini \& Buzzoni and a more recent update by Yi \& Yoon 2004)

Resolved color-magnitude (c-m) diagrams of stellar populations in M32 (Brown et al. 1998, 2000) have def1986) (see O'Connell 1999, , for an exhaustive review,

initely shown that even its relatively poor UV emission almost entirely arises from a fraction of hot HB stars, further complemented by a minority contribution from post-AGB PN nuclei. Still, facing the established interpretative scenario, one is left with at least three important issues that need to be assessed to understand the real nature of the UV-upturn phenomenon.

(i) The canonical evolutionary framework experienced in Galactic globular clusters naturally predicts a blue HB morphology only for old, metal-poor stellar populations (Chiosi et al. 1992; Renzini \& Fusi Pecci 1988). If this is the case for ellipticals too, then UV stars should represent the $Z \ll Z_{\odot}$ tail of a (supposedly) broad metallicity distribution seen to peak at much higher values, around solar abundance. Clearly, a more composite picture might be envisaged once one admits nonstandard models (i.e., including the effects of stellar rotation, helium mixing, differential mass loss, etc.) to account, in particular, for the well known "second parameter" dilemma (Sweigart 1997; Buonanno et al. 1997; Catelan et al. 2001; Recio-Blanco et al. 2006). However, this unconventional approach still suffers from a somewhat arbitrary fine-tuning of the key physical assumptions.

Electronic address: alberto.buzzoni@oabo.inaf.it,r.gonzalez@astrosmo.unan. 4 ..j $)_{\mathrm{x}}$ Hot HB stars might, nonetheless, also be nat-

${ }^{1}$ INAF - Osservatorio Astronomico di Bologna, Via Ranzani 1, urally predicted among super metal-rich stellar mod40127 Bologna (Italy)

${ }^{2}$ Centro de Radioastronomía y Astrofísica, Universidad Nacional Autónoma de México, 58190 Morelia, Michoacán (Mexico) els, as far as metal abundance (and the linked helium content) exceeds some critical threshold. Presumably, in this case mass-loss allows stars to reach 
the HB phase with a conveniently low external envelope, compared to the helium core mass (Dorman et al. 1993; Castellani et al. 1992; Yi et al. 1998; Buzzoni 1995; D'Cruz et al. 1996). Such "extreme HB" stars (EHB) have actually been observed, for example, in $\omega$ Cen (D'Cruz et al. 2000), NGC 6388 and NGC 6441 (Rich et al. 1997), and in some old Galactic open clusters as well, like NGC 6791 (Kaluzny \& Udalski 1992; Buson et al. 2006); they clearly remain the favorite candidates to explain the evolutionary framework of UVenhanced elliptical galaxies (Busso et al. 2005).

This hypothesis implies, however, a direct relationship between chemical abundance (modulating the helium core mass at the HB onset) and mass-loss efficiency (to suitably "peel off" the stellar envelope along the RGB). As a consequence, one has to expect the UV-to-optical color to be, eventually, one of the most quickly evolving features in the SED of elliptical galaxies (Park \& Lee 1997). In theory, the UV-upturn can fade by several magnitudes as the lookback time increases by a few Gyr, although the effect is still detectable at intermediate redshift $(z \sim 0.3)$ (Brown et al. 2003; Ree et al. 2006). Unfortunately, the evolutionary details are extremely model-dependent, and a strong UV excess could be triggered at ages as early as $\sim 6$ Gyr (Tantalo et al. 1996) or as late as $\gtrsim 15$ Gyr (Yi et al. 1998).

(iii) An established correlation seems to be in place between PN luminosity-specific rate and $(B-V)$ color for elliptical galaxies in the Virgo and Fornax clusters, and in the Leo group (Peimbert 1990; Hui et al. 1993). The sense is that reddest metal-rich systems display, at the same time, a stronger UV-upturn (Burstein et al. 1988) and a poorer PN population per unit galaxy luminosity (Buzzoni et al. 2006). If the $\mathrm{PN}$ event is the final fate for AGB stars at the end of their thermal pulsing phase (Iben \& Renzini 1983), then the relative deficiency of nebulae might be evidence of an incomplete (or fully inhibited) AGB evolution of low-mass stars under special environment conditions of the parent galaxy.

As a central issue in this discussion, it is clear therefore that a preeminent connection should exist between $U V$ excess and $A G B$ distinctive properties of stellar populations in early-type galaxies.

On account of the Fuel Consumption Theorem (Renzini \& Buzzoni 1986), a $1 \mathrm{M}_{\odot}$ star of solar metallicity enters its core He-burning phase with, at most, the equivalent of $0.43 \mathrm{M}_{\odot}$ of $\mathrm{H}$ to be spent as nuclear fuel. ${ }^{3}$ This means that, under quite general conditions, post-RGB evolution alone could easily account, in principle, for up to $3 / 4$ of the total bolometric luminosity of a galaxy stellar population (Buzzoni 1998). Whether this energy is eventually reduced (if stars loose their fuel before they burn it), or whether it is finally released in the form of ultraviolet or infrared photons, crucially depends on mass-loss and its impact along the entire red-giant

3 Under the most extreme hypothesis of no mass-loss, a $1 \mathrm{M}_{\odot}$ star with solar abundance $(Y, Z)=(0.28,0.02)$ starts its HB evolution with a total $\mathrm{He}$ amount of roughly $0.62 \mathrm{M}_{\odot}$, of which $\sim 0.47 \mathrm{M}_{\odot}$ are confined in the core (Sweigart \& Gross 1976) and $Y(1-0.47) \simeq 0.15 \mathrm{M}_{\odot}$ reside in the envelope. Metals amount to roughly $Z(1-0.47) \simeq 0.01 \mathrm{M}_{\odot}$ and, accordingly, fresh $\mathrm{H}$ is $0.37 \mathrm{M}_{\odot}$. Taking into account the nuclear rates (e.g., Cox \& Giuli 1968), the $\mathrm{H}+\mathrm{He}$ fuel provides at most the equivalent of $0.37+0.62 / 10 \simeq$ $0.43 \mathrm{M}_{\odot}$ of hydrogen. evolution. Hence, it is of special pertinence to constrain the relevant physical conditions that affect AGB evolution in favor of an earlier transition of HB stars towards high temperature and enhanced ultraviolet emission.

In this paper we would like to draw the reader's attention to a possibly new and powerful approach to the problem, that can find straightforward applications even to distant galaxies. As explained in Sec. 2, the method relies on surface-brightness fluctuation theory to safely tie infrared effective magnitudes (that can be determined for unresolved stellar populations), to stellar luminosity at the AGB tip (that cannot be directly observed in distant galaxies). We will show, in Sec. 3, that these results tightly correlate with the ultraviolet properties of "UV upturn" elliptical galaxies, allowing a self-consistent physical picture and a quite accurate diagnostic of the post-RGB evolution of their underlying stellar populations, including HB morphology and AGB deployment. Our results will be finally summarized and discussed in Sec. 4.

\section{INFRARED SURFACE-BRIGHTNESS FLUCTUATIONS AS AGB PROBES}

Tonry \& Schneider (1988) and Tonry (1991) first realized the potentially useful information about the composing stars hidden in the surface brightness fluctuations (SBFs) of galaxies with unresolved stellar populations. The problem has since received a more complete theoretical assessment by Buzzoni (1989, 1993, 2008); Cerviño et al. (2000, 2002), and Cerviño \& Luridiana (2006).

Briefly, the basic relationship upon which the theory relies is

$$
\frac{\sigma^{2}\left(L_{\mathrm{gal}}\right)}{L_{\mathrm{gal}}}=\frac{\sum \ell_{*}^{2}}{\sum \ell_{*}}=\ell_{\mathrm{eff}} .
$$

The 1.h. side of the equation links an observable quantity (namely, the relative variance of the galaxy surface brightness) with the theoretical second-order statistical moment of the composing stars. This quantity, in turn, traces the distribution of stellar luminosity $\left(\ell_{*}\right)$ for the whole population; it is also a natural output of any population synthesis code, and can easily be computed for different photometric bands and distinctive evolutionary phases of simple stellar populations (SSPs; see, e.g., the current theoretical databases of Buzzoni 1993; Worthev 1993; Blakeslee et al. 2001; Cerviño et al. 2002; Raimondo et al. 2005; Mouhcine et al. 2005; Cantiello et al. 2003).

The derived "effective" stellar luminosity, $\ell_{\mathrm{eff}}$, in eq. (11) has some important properties. (a) It can be derived from a fully observational procedure, without any supplementary theoretical assumptions. (b) It is an intrinsic distinctive parameter of the stellar aggregate, and its empirical measurement does not require any individual star to be (fully or partially) resolved. (c) It identifies an "effective" magnitude (i.e. $\bar{M}=-2.5 \log \ell_{\mathrm{eff}}+$ const) in a completely similar way and with the same photometric zero points as "standard" magnitudes, and it scales accordingly with distance and foreground screen reddening.

Being statistically representative of the stellar system as a whole, the effective magnitude cannot be physically associated to any specific star or stellar groups along the 


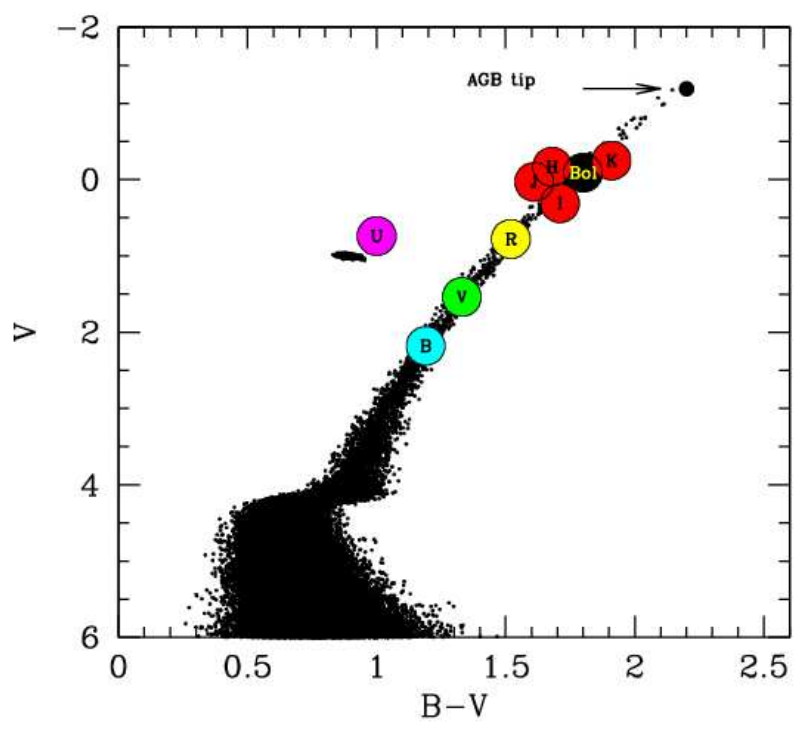

FIG. 1.- An illustrative $V$ vs. $B-V$ synthetic c-m diagram for a 15 Gyr SSP of solar metallicity and Salpeter IMF, with big dots identifying the different "effective contributors" (i.e., the appropriate magnitude $M \equiv \bar{M}$ ) at various photometric bands (and to bolometric luminosity, as well). Although the different effective magnitudes cannot univocally be attributed to any $B-V$ color, one could let the prevailing stellar contributors to bolometric, infrared, and visual fluctuations arbitrarily coincide with the corresponding RGB location in the c-m diagram. This is not the case for the $U$ band, where the effective magnitude comes from a more entangled mix of $\mathrm{HB}$, red giants, and bright main sequence (MS) stars about the turn-off region of the diagram.

c-m diagram of a stellar aggregate; nonetheless, it can be instructive to identify stars with $\bar{M}$ luminosity as the "prevailing" group tracing the whole population in the different photometric bands. This is shown in Fig. 1 for the illustrative case of a 15 Gyr SSP with solar metallicity and Salpeter IMF. Because of the quadratic $\ell_{*}$ dependence of the summation in the numerator of eq. (1), the effective magnitude at any optical/infrared band is highly sensitive to the giant stars and only marginally responds to a shift in the SSP "mass leverage" caused by a change in the IMF slope (Buzzoni 1993). Likewise, as probe of the brightest stars in a population at a given wavelength, the effective magnitude is relatively insensitive to any underlying older component in the case of composite stellar systems. ${ }^{4}$

It is evident from Fig. 1 that near-infrared (near-IR) magnitudes closely trace the bright-end tail of the SSP luminosity function. In particular, one can notice that $\bar{K}$ is potentially the best tracer of the SSP tip stellar luminosity $\left(K_{\text {tip }}\right)$, as both quantities are expected to depend in quite the same way on the overall distinctive parameters of the stellar population, including age, metallicity, $I M F$, and mass-loss. ${ }^{5}$ This is shown in Fig. 2, where

\footnotetext{
4 This fact has been observationally confirmed through the comparison between SBF measurements in early-type galaxies and Magellanic star clusters (see González et al. 2004; González-Lópezlira et al. 2005).

${ }^{5}$ In spite of probing a relative minority of bright giant stars, the infrared effective magnitude is a fully robust characteristic parameter of a stellar population, in the case of galactic mass scales. From a statistical point of view, in fact, taking the reference SSP of Fig. 1 as representative of the elliptical galaxy stellar population, one has to expect about $9 \times 10^{7}$ "effective contributors" (in
}

we compare the $\bar{K}$ vs. $K_{\text {tip }}$ relationship for a full collection of SSP models from the Buzzoni (1989) and the updated Charlot \& Bruzual (CB07 Bruzual 2007) synthesis codes. ${ }^{6,7}$

The correlation appears quite robust, in spite of the extremely wide range explored for the population parameters, and the different input physics adopted by the two sets of theoretical models.

Formal fits to the whole sets of models yield:

$$
\left\{\begin{array}{rr}
K_{\text {tip }}=0.75 \bar{K}-3.15 & \sigma= \pm 0.12 \mathrm{mag} \\
& \text { (Buzzoni 1989) } \\
K_{\text {tip }}=0.74 \bar{K}_{s}-3.07 & \sigma= \pm 0.25 \mathrm{mag} \\
& \text { (Bruzual 2007) }
\end{array}\right.
$$

\subsection{AGB completion in young stellar populations: the case of Magellanic star clusters}

A comparison with real stellar populations extending across the widest range of evolutionary parameters would clearly be the ideal test for the envisaged theoretical picture. The Magellanic Clouds (MCs) stand out as striking candidates, in this regard, with the age of their star clusters spanning over four orders of magnitude, from a few Myr up to $\sim 10^{10}$ yr. In addition, their relatively close distance allows both fluctuation luminosity and AGB/RGB luminosity tip to be directly measured on resolved c-m diagrams.

Near-IR SBFs for a sample of $191 \mathrm{MC}$ star clusters have been obtained by González et al. (2004, 2005) using $K_{s}$-band data from the Two Micron All Sky Survey (2MASS, Skrutskie et al. 1997). To reduce stochastic effects due to small-number stellar statistics along fast evolutionary phases, in their study these authors assembled seven "super-clusters", by homogeneously coadding several objects for each age class according to the Searle et al. (1980, , SWB) classification scheme.

The procedure to obtain the SBFs for these data has been extensively described before (González et al. 2004; Mouhcine et al. 2005); briefly, direct summation of bright stars provided the numerator of the r.h. fraction of eq. (11), while total luminosity (at the denominator of the equation) was safely estimated from integrated photometry of each supercluster mosaic, to include the contribution of faint, unresolved stars. Following Lee et al. (1993), for the present work we have determined the tip luminosity of the AGB+RGB by convolving the luminosity function of each supercluster with a Sobel edgedetection filter (the zero-sum kernel $[-2,0,+2]$ ). The tip

the statistical definition of Buzzoni 1993) to the $K$-band SBF for a galaxy of $10^{11} \mathrm{M}_{\odot}$. This number further increases to about $10^{8}$ for $H$-band contributors, and to $\sim 3 \times 10^{8}$ for the $J$ band SBF.

6 The $K_{s}$ band is a variant of the standard $K$ filter, sometimes preferred for its reduced background noise (see Persson et al. 1998, for specific details). Both filters have similar effective wavelength and photometric zero points, so that $K=K_{s}$ within a typical $\pm 0.03 \mathrm{mag}$ uncertainty, as we directly verified in our tests.

7 For the CB07 models, the fluctuation luminosity has been computed via eq. (1) from the original isochrone set, while $K_{\text {tip }}$ is just read as the absolute magnitude of the brightest stellar type in the AGB+RGB phases. 

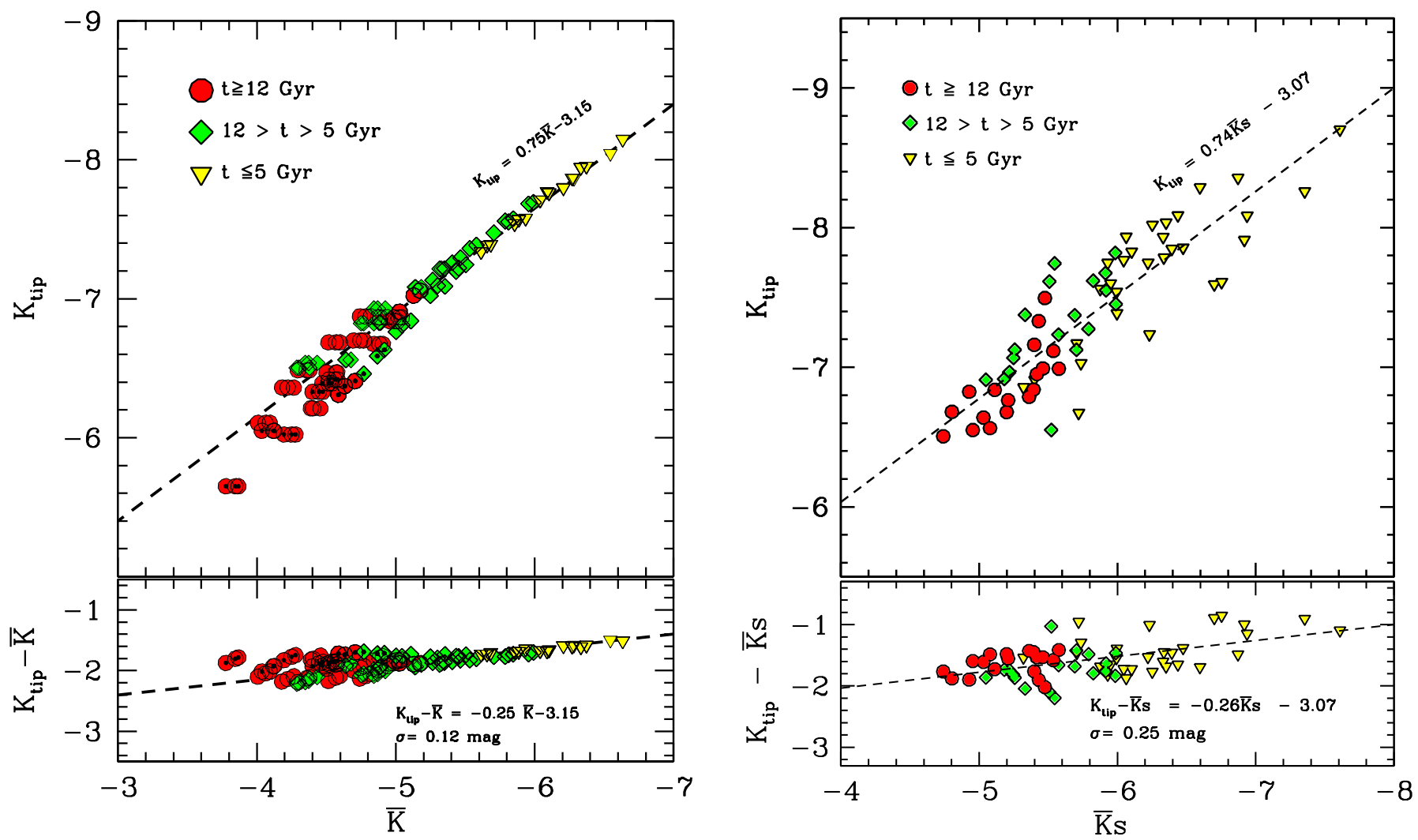

FIG. 2.- Theoretical relationship between near-IR effective magnitude and AGB+RGB tip luminosity, for a full collection of SSP models from Buzzoni (1989, , left panel), and Charlot \& Bruzual (Bruzual 2007, , right panel). Population ages span from 1 Gyr (upper right) to $18 \mathrm{Gyr}$ (lower left); metallicity ranges between $0.0001 \leq Z \leq 0.05$. The Buzzoni models explore a Reimers (1975) mass-loss parameter $\eta=0.3$ and 0.5 for a Salpeter IMF, while the Charlot \& Bruzual calculations adopt the mass-loss rates derived by Marigo \& Girardi (2007) and use a Chabrier (2003) IMF. Dashed lines indicate fits to all points. Besides the overall agreement between the two synthesis codes, one has to notice a larger scatter around the mean relation for the CB07 models, mainly due to a different response to SSP metallicity at younger ages.

TABLE 1

PARAMETERS OF MC STELlar "SUPERClusters"

\begin{tabular}{lcccc}
\hline \hline $\begin{array}{c}\text { SWB } \\
\text { type }\end{array}$ & $\begin{array}{c}\bar{M}_{K_{s}} \\
{[\mathrm{mag}]}\end{array}$ & $\begin{array}{c}M_{K_{\mathrm{tip}}} \\
{[\mathrm{mag}]}\end{array}$ & $\log$ age $(\mathrm{yr})$ & $\log Z$ \\
\hline pre & $-7.70 \pm 0.40$ & $-8.7 \pm 0.2$ & $6.4 \pm 0.3$ & $-0.3 \pm 0.2$ \\
I & $-8.85 \pm 0.12$ & $-9.8 \pm 0.2$ & $7.0 \pm 0.3$ & $-0.3 \pm 0.2$ \\
II & $-7.84 \pm 0.28$ & $-8.9 \pm 0.2$ & $7.5 \pm 0.3$ & $-0.3 \pm 0.2$ \\
III & $-7.45 \pm 0.24$ & $-8.3 \pm 0.2$ & $8.0 \pm 0.3$ & $-0.1 \pm 0.2$ \\
IV & $-7.51 \pm 0.18$ & $-8.4 \pm 0.2$ & $8.5 \pm 0.3$ & $-0.8 \pm 0.2$ \\
V & $-6.69 \pm 0.20$ & $-8.0 \pm 0.2$ & $9.0 \pm 0.3$ & $-0.6 \pm 0.2$ \\
VI & $-6.21 \pm 0.24$ & $-7.6 \pm 0.2$ & $9.5 \pm 0.3$ & $-1.0 \pm 0.2$ \\
VII & $-4.92 \pm 0.38$ & $-7.2 \pm 0.2$ & $9.9 \pm 0.3$ & $-1.4 \pm 0.2$ \\
\end{tabular}

Note. - Col. (2): $\bar{M}_{K}$ from González et al. (2004, 2005). Col. (4): Ages from Frogel et al. (1990), corrected to the Large Magellanic Cloud distance modulus, $(\mathrm{m}-\mathrm{M})_{\circ}=18.5$, as in Mouhcine et al. (2005); the age of the pre-SWB supercluster is also from González et al. (2004). Col. (5): Metallicities from Cohen (1982), for cluster types pre-SWB, I, and II, and from Frogel et al. (1990) for later types.

luminosity was identified with the peak of the filter response function, after checking that there is indeed an important count discontinuity at that location in the luminosity function; the error in the measurement equals the width of the histogram bin. We list in Table 1 the $K_{s}$-band absolute fluctuation magnitude, $K_{s}$ absolute tip magnitude, age, and metallicity of the MC superclusters.

Again, a tight relationship between $K_{\text {tip }}$ and effective

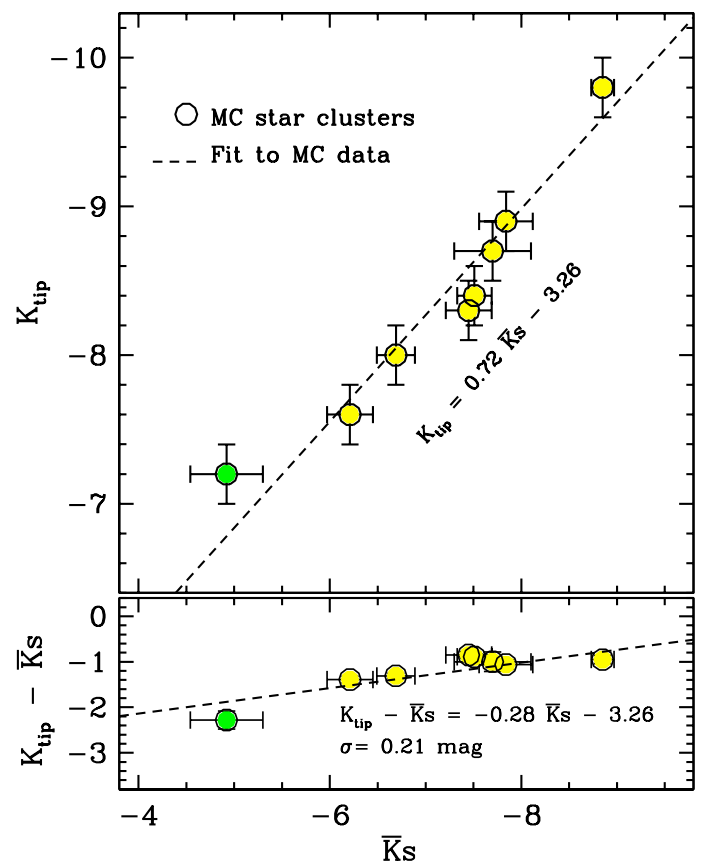

Fig. 3.- Absolute $K_{\text {tip }}$ vs. $\overline{K_{s}}$ magnitude relationship for Magellanic Cloud star clusters. Big dots locate the average position for each "supercluster" of Table 1, i.e. a co-addition of several individual systems belonging to homogenous Searle et al. (1980) age classes. The dashed line is the fit to the data, as displayed on the plot. Color code for markers is like in Fig. 2. 


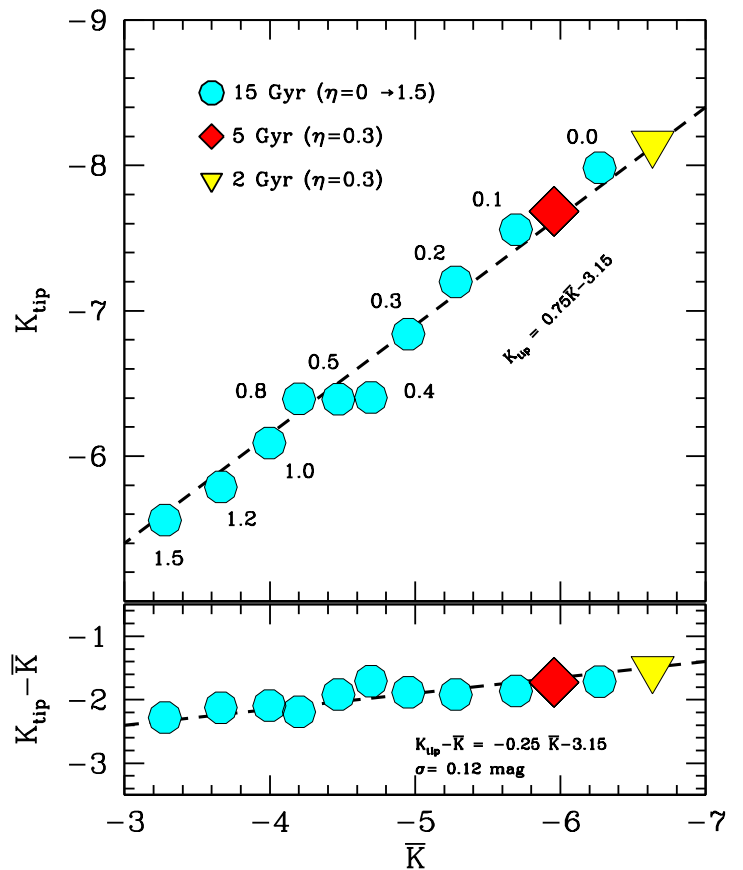

FIG. 4.- Theoretical relationship between $K_{\text {tip }}$ and $\bar{K}$ with varying mass loss efficiency along red-giant evolution. Big dots trace the change in a 15 Gyr old SSP of solar metallicity (Buzzoni 1989), with the increase of Reimers mass-loss parameter $\eta$ from 0 to 1.5 , as labelled on the plot. Note that a reduced mass loss $(\eta \rightarrow 0)$ leads old SSPs to fully deploy the AGB and closely resemble much younger 2-5 Gyr old SSPs (triangles and diamond markers on the plot) with $\eta=0.3$ and otherwise same distinctive parameters.

$\bar{K}$ magnitudes is in place, as shown in Fig. 3, along the entire age range (and the corresponding metallicity drift, see column 5 in Table 1). A fit to the MC data (dashed line in the figure) provides:

$$
K_{\text {tip }}=0.72 \bar{K}_{s}-3.26
$$

with a data scatter $\sigma= \pm 0.21$ mag across the fitting line. Note that this perfectly compares, within the statistical uncertainty of the observations, with both eqs. (2); this is true even beyond the nominal age range of the theoretical relations since, for instance, the pre-SWB and the SWB type I MC superclusters are too young to even sport any standard AGB or RGB phase!

\subsection{Mass-loss and post-RGB evolution in evolved stellar populations}

The link between $\bar{K}$ and $K_{\text {tip }}$ can be regarded as a much more deeply intrinsic property of SSPs, not exclusively related to age (or metallicity) evolution. Of course, our method cannot remove, by itself, any age/metallicity degeneracy, that makes integrated photometric properties of young metal-rich stellar systems closely resemble those of older metal-poor ones (e.g. Renzini \& Buzzoni 1986; Worthey 1994). However, as far as old stellar populations are concerned, the unbiased and modelindependent estimate of the tip stellar luminosity, even in unresolved stellar populations, provides an additional and powerful tool to quantitatively size up important effects, like the mass-loss impact on red-giant evolution.

Relying on the Reimers (1975) standard $\eta$ parameterization, Fig. 4 gives an instructive example of how massloss can modulate both $K_{\text {tip }}$ and $\bar{K}$. In the figure we explore the behavior of an old (15 Gyr) SSP reference model (big dots in the plot), along a full variety of massloss scenarios, spanning from a virtually vanishing stellar wind (i.e. $\eta=0)$, up to a violent mass-loss rate $(\eta \rightarrow 1.5)$ capable of fully wiping out the external envelope of stars while on the RGB phase. Similarly to Fig. 3, we still report the same clean relationship between $K_{\text {tip }}$ and $\bar{K}$, with old AGB-enhanced models $(\eta \rightarrow 0)$ closely resembling, all the way, young (2-5 Gyr) standard $(\eta \simeq 0.3)$ populations in the upper right corner of the panel.

Again, according to the trend of the $15 \mathrm{Gyr}$ model sequence vs. $\eta$ we can propose the following parametric equations:

$$
\left\{\begin{array}{l}
K_{\text {tip }} \simeq 1.7 \eta-7.7 \\
\bar{K} \simeq 2.1 \eta-5.9 .
\end{array}\right.
$$

Curiously enough, note from the equation set that $K_{\text {tip }}$ seems slightly less sensitive to $\eta$ than $\bar{K}$. As we will see in a moment, such a smaller variation range for $K_{\text {tip }}$ stems from the "bottoming out" effect of the RGB tip, that eventually replaces the AGB in providing the brightest stars in a SSP when mass-loss increases to $\eta \gg 0.5$.

The now established importance of mass-loss in old stellar populations dominated by stars with $M_{*} \simeq 1 \mathrm{M}_{\odot}$ was first emphasized in a series of important theoretical contributions in the early seventies (Castellani \& Renzini 1968; Iben \& Rood 1970; Castellani et al. 1970); this was urged by the intervening observational evidence that a mass spread was needed to reproduce HB and AGB morphology in Galactic globular clusters (Demarque et al. 1972; Renzini 1977; Iben \& Renzini 1983). To consistently match the observed AGB tip in local globular clusters, for instance, Fusi Pecci \& Renzini (1976) suggested a fine-tuning value of $\eta \simeq 0.4 \pm 0.1$. This calibration for old Pop II stars might however not so straightforwardly be extended, a priori, to more metal-rich environments, as one could realistically expect mass-loss efficiency to depend on (increase with?) metallicity.

Depending on mass-loss strength, standard stellar evolution theory basically features three characteristic scenarios that constrain post-RGB evolution of low-mass stars (see Fig. 5). Under different physical conditions and to a different extent, each one of these cases eventually leads to the formation of hot stars, thus potentially supplying an important contribution to the integrated ultraviolet luminosity of a galaxy.

(a) For $\eta \lesssim 0.4$, models tell us that at the end of RGB evolution, low-mass stars ignite helium in a degenerate core (the so-called "helium flash"). A bright AGB evolution has to be expected, reaching or even surpassing the RGB tip luminosity. If thermal pulses are set on $(\eta \lesssim 0.3)$, stars end up loosing the external envelope and generating a planetary nebula (Iben \& Renzini 1983). In this case, hot post-AGB nuclei of nebulae easily exceed an effective temperature of $30000 \mathrm{~K}$ (Schönberner 1983).

(b) If $\eta \gtrsim 0.4$, the PN event is aborted (Buzzoni et al. 2006), and AGB evolution is partially or fully inhibited (Greggio \& Renzini 1990; Castellani \& Tornambé 1991; Dorman et al. 1993). In this case, the brightest stars (both in bolometric luminosity and in the infrared) belong to the RGB tip, and they settle on the HB with a He core mass close or slightly exceed- 
TABLE 2

Summary of RELEVANT DATA FOR THE ELLIPTICAL-GALAXY SAMPLE

\begin{tabular}{|c|c|c|c|c|c|c|c|c|}
\hline NGC & $\begin{array}{c}\log \sigma_{\mathrm{v}} \\
{\left[\mathrm{km} \mathrm{s}^{-1}\right]}\end{array}$ & $\begin{array}{l}\mathrm{Mg}_{2} \pm \sigma \\
{[\mathrm{mag}]}\end{array}$ & $\begin{array}{c}(B-V)_{o} \\
{[\mathrm{mag}]}\end{array}$ & $\begin{array}{c}\log \alpha \\
\mathrm{N}_{\mathrm{PN}} \mathrm{L}_{\text {gal }}^{-1} \\
\end{array}$ & $\begin{array}{c}(1550-V)_{o} \\
{[\mathrm{mag}]}\end{array}$ & $\begin{array}{c}\overline{F 160 W} \pm \sigma \\
{[\mathrm{mag}]}\end{array}$ & $\begin{array}{c}\bar{K}_{s} \pm \sigma \\
{[\mathrm{mag}]}\end{array}$ & $\begin{array}{c}H \beta \pm \sigma \\
{[\AA]}\end{array}$ \\
\hline 221 & $1.90 \pm 0.03$ & $0.198 \pm 0.007$ & 0.88 & $-6.77_{0.31}^{0.18}$ & $4.50 \pm 0.17$ & $-5.28 \pm 0.10$ & $-5.95 \pm 0.14$ & $2.30 \pm 0.05$ \\
\hline 224 & $2.27 \pm 0.03$ & $0.324 \pm 0.007$ & 0.95 & $-6.94_{0.22}^{0.15}$ & $3.51 \pm 0.17$ & $-4.46 \pm 0.09$ & $-5.69 \pm 0.14$ & $1.66 \pm 0.07$ \\
\hline 1316 & $2.38 \pm 0.08$ & $0.265 \pm 0.023$ & 0.87 & $-7.50_{0.07}^{0.07}$ & $5.0 \pm 0.2$ & $-5.39 \pm 0.19$ & $\ldots$ & $2.20 \pm 0.07$ \\
\hline 1379 & $2.11 \pm 0.13$ & $0.269 \pm 0.006$ & 0.88 & $\ldots$ & $3.01 \pm 0.10$ & $-5.11 \pm 0.19$ & $-5.75 \pm 0.12$ & $1.70 \pm 0.09$ \\
\hline 1387 & $\ldots$ & $\ldots$ & 0.98 & $\ldots$ & $2.16 \pm 0.05$ & $-5.4 \pm 0.8$ & $-5.77 \pm 0.10$ & $\ldots$ \\
\hline 1389 & $2.12 \pm 0.06$ & $0.236 \pm 0.002$ & 0.90 & $\ldots$ & $3.38 \pm 0.09$ & $-5.16 \pm 0.20$ & $-6.35 \pm 0.13$ & \\
\hline 1399 & $2.52 \pm 0.03$ & $0.357 \pm 0.007$ & 0.95 & $-7.30_{0.07}^{0.07}$ & $2.05 \pm 0.17$ & $-4.55 \pm 0.16$ & $-5.28 \pm 0.15$ & $1.41 \pm 0.08$ \\
\hline 1404 & $2.39 \pm 0.03$ & $0.344 \pm 0.007$ & 0.95 & $\ldots$ & $3.30 \pm 0.17$ & $-4.76 \pm 0.21$ & $-5.53 \pm 0.10$ & $1.58 \pm 0.08$ \\
\hline 3379 & $2.33 \pm 0.03$ & $0.329 \pm 0.007$ & 0.94 & $-6.77_{0.03}^{0.03}$ & $3.86 \pm 0.17$ & $-4.70 \pm 0.14$ & $-5.43 \pm 0.17$ & $1.46 \pm 0.16$ \\
\hline 3384 & $2.20 \pm 0.11$ & $0.296 \pm 0.014$ & 0.91 & $-6.42_{0.10}^{0.10}$ & $3.9 \pm 0.2$ & $-4.82 \pm 0.22$ & $\ldots$ & $2.05 \pm 0.11$ \\
\hline 4278 & $2.45 \pm 0.03$ & $0.293 \pm 0.007$ & 0.90 & $\ldots$ & $2.88 \pm 0.17$ & $-4.49 \pm 0.22$ & $\ldots$ & $1.37 \pm 0.03$ \\
\hline 4374 & $2.48 \pm 0.03$ & $0.323 \pm 0.007$ & 0.94 & $-6.77_{0.10}^{0.10}$ & $3.55 \pm 0.17$ & $\ldots$ & $-5.71 \pm 0.25$ & $1.70 \pm 0.04$ \\
\hline 4406 & $2.42 \pm 0.03$ & $0.330 \pm 0.007$ & 0.90 & $-6.89_{0.10}^{0.10}$ & $3.72 \pm 0.17$ & $\ldots$ & $-5.74 \pm 0.12$ & $1.61 \pm 0.16$ \\
\hline 4472 & $2.49 \pm 0.03$ & $0.331 \pm 0.007$ & 0.95 & $-7.16_{0.10}^{0.10}$ & $3.42 \pm 0.17$ & $-4.64 \pm 0.11$ & $-5.64 \pm 0.13$ & $1.52 \pm 0.14$ \\
\hline 4486 & $2.60 \pm 0.03$ & $0.303 \pm 0.007$ & 0.93 & $-7.10_{0.10}^{0.1}$ & $2.04 \pm 0.17$ & $\ldots$ & $\ldots$ & $1.38 \pm 0.04$ \\
\hline 4552 & $2.44 \pm 0.03$ & $0.346 \pm 0.007$ & 0.94 & $\ldots$ & $2.35 \pm 0.17$ & $\ldots$ & $-5.86 \pm 0.13$ & $1.65 \pm 0.04$ \\
\hline 4621 & $2.41 \pm 0.03$ & $0.355 \pm 0.007$ & 0.92 & $\ldots$ & $3.19 \pm 0.17$ & $\cdots$ & $-5.59 \pm 0.21$ & $1.43 \pm 0.11$ \\
\hline 4649 & $2.56 \pm 0.03$ & $0.360 \pm 0.007$ & 0.95 & $-7.22_{0.10}^{0.1}$ & $2.24 \pm 0.17$ & $\ldots$ & $\ldots$ & $1.26 \pm 0.04$ \\
\hline
\end{tabular}

Note. - Cols. (2) and (3). $\log \sigma$ and $\mathrm{Mg}_{2}$ index from Burstein et al. (1988), except for NGC 1316, 1379, 1389, 3384, that are averages from the Hyperleda database (Paturel et al. 2003). Col. (4). ( $B-V$ )o from the RC3 Catalogue (de Vaucouleurs et al. 1991). Col. (5). Log $\alpha$ from Buzzoni et al. (2006). Col. (6) (1550-V) color from Burstein et al. (1988), except for NGC 1379, 1387, 1389, and 1404; these were scanned from Lee et al. (2005) with the Dexter package (Demleitner et al. 2001), and transformed according to the Colina et al. (1996) calibration scale. Col. (7). $\bar{M}_{F 160 W}$ from Jensen et al. (2003). Col. (8). $\bar{M}_{K s}$ from Liu et al. (2002) (assuming the Cepheids distance modulus for the Fornax cluster), excepting NGC 221, 224, 3379, and 4374, that are from Pahre \& Mould (1994), and NGC 4406 and 4472, from Jensen et al. (1998). Col. (9). H $\beta$ index from Jensen et al. (2003), excepting NGC 4278, 4374, 4486, 4649, 4552, estimated from Kobayashi \& Arimoto (1999), and NGC 4621, derived from Kuntschner et al. (2001).

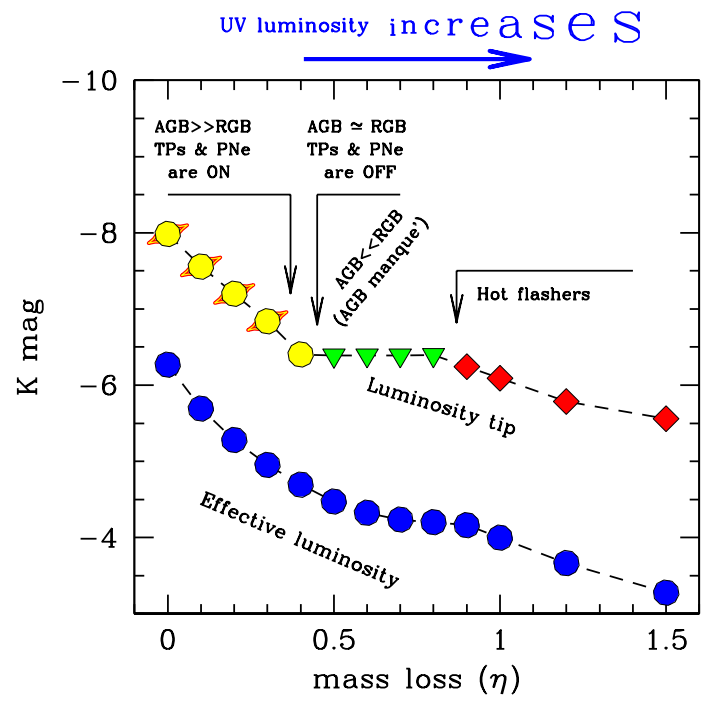

FIG. 5.- Theoretical $\bar{K}$ and $K_{\text {tip }}$ relationship with increasing mass-loss rate along red-giant evolution. The illustrative case of a 15 Gyr old SSP of solar metallicity, after Buzzoni (1989), is considered. Note that, for $\eta \lesssim 0.4$, the AGB luminosity tip is brighter than the RGB and PNe are produced; if mass loss increases, then AGB luminosity is further reduced (and PN formation correspondingly thwarted), until stars at the RGB tip begin to dominate as the brightest objects in the population $(\eta \gtrsim 0.5)$. For even higher mass-loss rates $(\eta \gtrsim 0.8)$ stars undergo incomplete RGB evolution, leaving the branch "midway" and igniting helium at a higher effective temperature (hot flashers). ing 0.47 $\mathrm{M}_{\odot}$ (e.g., Sweigart \& Gross 1976; Seidel et al. 1987; Charbonnel et al. 1996; Piersanti et al. 2004). For slightly increasing mass-loss efficiency, the stellar envelope becomes thinner and thinner, and the hot internal core is unveiled. If this is the case, stars likely settle at high effective temperature $\left(T_{\text {eff }} \gg 20000 \mathrm{~K}\right)$ and originate a blue $\mathrm{HB}$ morphology.

(c) As a final case, for an even stronger mass-loss $(\eta \gtrsim 0.8)$, the stellar envelope might be wiped out well before the full completion of the RGB phase. As a consequence, standard $\mathrm{HB}$ and AGB evolution would be fully inhibited; stars would be left "midway" along the RGB, with their rapidly exhausting H-shell emerging at ever shallower stellar layers. Such "hot flasher" stars would therefore postpone the He ignition by moving straight to the high-temperature region of the $\mathrm{c}-\mathrm{m}$ diagram, as is likely observed, indeed, in the cluster NGC 2808 (Sweigart et al.|2002; Castellani et al.|2006).

In terms of UV contribution, cases (a), (b), and (c) represent a sequence of increasing energy budget, as a prevailing fraction of stellar nuclear fuel is more efficiently burnt at high effective temperature. The outlined scenario has, of course, to be regarded as a general scheme, where the $\eta$ thresholds discriminating among the different evolutionary regimes slightly depend on SSP age (through the MS Turn Off mass). While the illustrative case of Fig. 5 refers to a 15 Gyr SSP, one could easily verify that the overall trend for $K$ luminosity is maintained, for instance for a 10 Gyr population, by just "shrinking" the $\eta$-scale of the plot by a factor of $\sim 1.5 \times$; this means, 


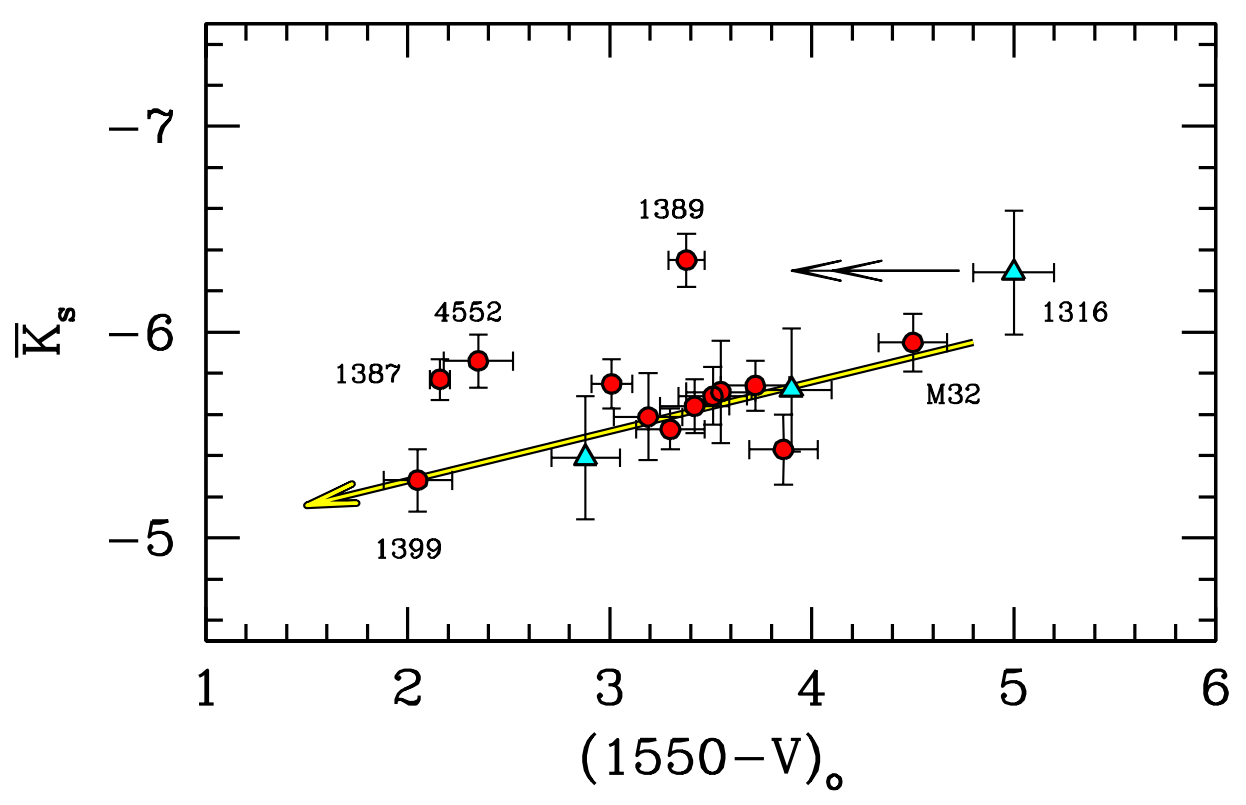

FIg. 6.- Absolute fluctuation magnitudes vs. ultraviolet color $(1550-V)$ for the galaxy sample of Table 2. Both $\overline{K_{s}}$ and $(1550-V)$ have been duly corrected for Galactic reddening. Triangles mark galaxies with $\overline{K_{s}}$ extrapolated from $\overline{F 160 W}$, as explained in footnote 8 Note the outlying cases of NGC 1387, NGC 4552, and NGC 1389 with an infrared effective magnitude that is $\sim 0.7$ mag too bright for their UV excess. The relevant case of the merger radio-galaxy NGC 1316 (Fornax A) is also singled out in the plot, where the arrow indicates that a "bluer" $(1550-V)$ color might be more appropriate for this galaxy (with a more negligible impact on $\bar{K}_{s}$, though), as a consequence of a strong internal absorption due to the observed presence of dust lanes. Excluding these controversial objects (discussed in more detail in Sec. 4), the data sample correlates fairly well ( $\rho=-0.75$, see arrow), indicating a less deployed AGB for UV-enhanced galaxies.

for example, that $A G B$-manqué stars are produced in a 10 Gyr scenario for $\eta \gtrsim 0.6$.

\section{UV UPTURN AND AGB DEPLOYMENT IN ELLIPTICAL GALAXIES}

In order to assess the relevance of the previous theoretical framework to the appearence and strength of the "UV-upturn" phenomenon in ellipticals, it is convenient to parameterize our analysis in terms of the $(1550-V)$ color, that is a measure of the galaxy emission around the $1550 \AA$ region vs. the Johnson visual band, as originally defined by Burstein et al. (1988). To this end, the Burstein et al. (1988) IUE galaxy sample has been taken as a reference; we supply in Table 2, for each object, the infrared effective magnitudes from Jensen et al. (2003), Liu et al. (2002), Pahre \& Mould (1994), and Jensen et al. (1998) and, when available, further relevant pieces of information, like stellar velocity dispersion, integrated $(B-V)$ color, $\mathrm{H} \beta$ and $\mathrm{Mg}_{2}$ Lick indices, and the specific PN rate per unit galaxy luminosity (from Buzzoni et al. 2006).

As a first relevant clue in our analysis, Fig. 6 is suggestive of a trend among most ellipticals, with UV-enhanced galaxies being about one mag fainter in $K_{s}$ effective magnitude (some 0.7 mag in the inferred $K_{\text {tip }}$ ), compared to UV-poor systems. ${ }^{8}$ On the plot one should report, however, a couple of outliers, displaying a brighter $\bar{K}$ mag-

\footnotetext{
${ }^{8}$ In order to increase the displayed galaxy sample on the different plots that involve infrared photometry, for some objects we derived the $K$-band effective luminosities based on the $F 160 \mathrm{~W} \equiv H$ photometry only assuming, empirically from Table $2,\left\langle\bar{H}-\bar{K}_{s}\right\rangle=+0.9$, with a conservative error bar of \pm 0.3 mag. The extrapolated $\bar{K}$ mag data are displayed with a different marker on the plots.
}

nitude (or, alternatively, a "bluer" $(1550-V)$ color). These include NGC 1387 and NGC 1389 in the Fornax cluster, and NGC 4552 in Virgo. We will be back to these objects in the next section, for a brief discussion. In addition, we have to mention that the "merger" radio-galaxy NGC 1316 (Fornax A) exhibits visible dust lanes that, when accounted for, may lead to an intrinsically "bluer" (1550 - $V$ ) color, although with possibly negligible effects on the $K \mathrm{SBF}$ magnitude.

After excluding these controversial objects, the data tend to display a fairly good correlation $(\rho=-0.75)$ that, also recalling eq. (4), we can approximate with

$$
\Delta \bar{K}_{s} \simeq 1.4 \Delta K_{\mathrm{tip}} \simeq-0.2 \Delta(1550-V) .
$$

If we entirely ascribe the dimming in red-giant tip luminosity suggested by Fig. 6 to a mass-loss effect, then eq. (4) implies a spread $\Delta \eta \simeq 0.4$ among the galaxy population, with UV-enhanced ellipticals requiring a Reimers parameter $\eta \simeq 0.3-0.4$, very similar to what Fusi Pecci \& Renzini (1976) derived, empirically, for the Galactic globular clusters. According to Fig. 5, this confirms that, overall, the AGB is deployed in luminosity slightly above the RGB tip, so that the brightest stars in ellipticals should indeed always be genuine AGB stars.

There is an obvious caveat, though, in this simplified picture. If a standard monolithic scenario is assumed for early-type galaxy formation, then gravitational collapse of the primeval gas clouds should have proceeded over a free-fall timescale $\tau_{\text {ff }} \propto \sigma_{v}^{-1} \propto \mathrm{M}_{\text {gal }}^{-1 / 2}$ (e.g., Larson 1974), leading to a mass-age-metallicity relation for early-type galaxies, where more massive galaxies are both older and more metal-rich than less massive ones (e.g., Pahre et al. 1998). The combined action of age and metallicity plays 


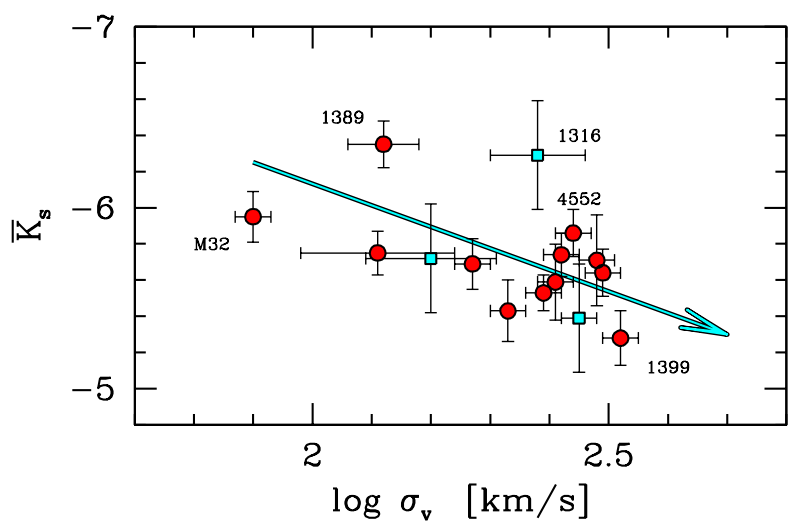

FIG. 7.- Absolute fluctuation magnitudes vs. velocity dispersion for the galaxy sample of Table 2 . Squares mark galaxies with $\overline{K_{s}}$ extrapolated from $\overline{F 160 W}$, as explained in footnote 8 .

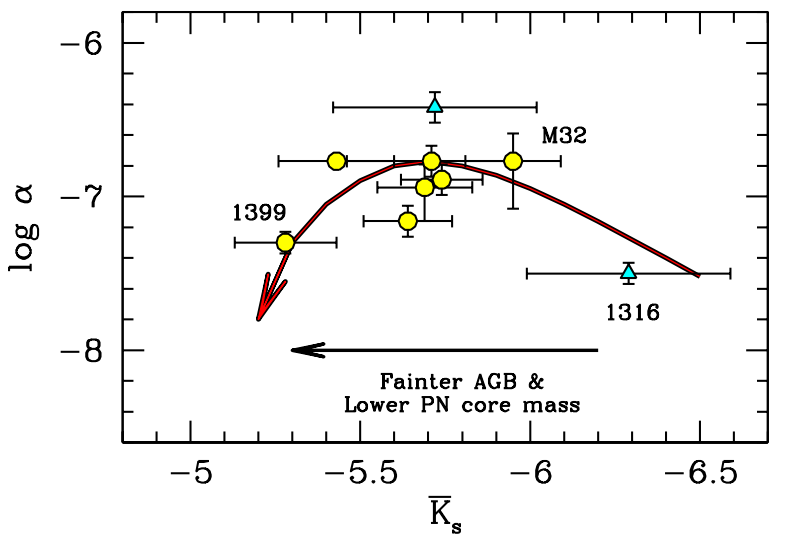

FIG. 8.- The luminosity-specific PN number $(\alpha)$ vs. effective $K$ luminosity for the galaxy sample of Table 2. The triangles mark galaxies with $\bar{K}_{s}$ inferred from $\overline{F 160 W}$ (see footnote 8). Note that a fainter AGB roughly correlates with a scantier PN stellar population among old/metal-rich (UV-enhanced) ellipticals, as expected from the long post-AGB to PN nucleus transition time, combined with the (shorter) evaporation timescale of the ejecta (compare the sketch on the plot with Fig. 15 in Buzzoni et al. 2006, , for a full discussion). A correspondingly low PN rate per unit galaxy luminosity is also to be expected, on the other hand, for younger stellar populations, as in the case of NGC 1316, as a consequence of higher stellar masses and a reduced PN nuclear lifetime.

an important role to modulate the red-giant luminosity tip, as the AGB is "naturally" brighter among young or metal-rich SSPs (see, again, Fig. 3).

The complex interplay of the different galaxy physical properties is well depicted in Figs. 7 and 8. For one, as Burstein et al. (1988) had already shown, the UV upturn seems to appear among the most massive and metal rich ellipticals (i.e., there is a positive correlation with $\sigma_{v}$ and the $\mathrm{Mg}_{2}$ index, respectively). Figure 7 indicates for these galaxies also a less extended AGB, as for a lower stellar core-mass distribution. This feature is likewise expected to directly affect the PN rate per unit galaxy luminosity (the so-called " $\alpha$ " index, see Fig. 8); indeed, there is a scantier PN stellar population among old/metal-rich (UV-enhanced) ellipticals (Ferguson \& Davidsen 1993; Buzzoni et al. 2006).

\subsection{Constraints on HB morphology}

Observations of local star samples show that the $\mathrm{H} \beta$ index reaches its maximum strength for A-type stars in the temperature range $\mathrm{T}_{\text {eff }} \simeq 8000 \rightarrow 10000 \mathrm{~K}$ (Buzzoni et al. 1994). Given this selected sensitivity, synthesis models of old SSPs (Buzzoni et al. 1994; Maraston et al. 2001) predict the integrated index to be strongly enhanced (by roughly $0.8 \AA$ or more) in the presence of a broad color-extended HB, as observed for most metal-poor Galactic globular clusters. This important piece of information could therefore usefully complement the more extreme $(1550-V)$ color in the analysis of $\mathrm{HB}$ morphology for unresolved stellar populations in distant galaxies.

The situation is summarized in Fig. 9, where elliptical galaxy data are compared with three illustrative cases of 15 Gyr SSPs with slightly supersolar metallicity $([\mathrm{Fe} / \mathrm{H}]=+0.3)$, and different ranges of $\mathrm{HB}$ temperature distribution. In particular, in the figure we account for (i) a red HB (RHB) morphology, that is, a clump of red stars very close to the RGB location, mimicking the real case of metal-rich Galactic globular clusters, like 47 Tuc; (ii) an intermediate HB (IHB) morphology, with a broad (roughly bell-shaped) temperature distribution peaked about the A-stars' temperature range and extending up to $T_{\text {eff }} \sim 12000 \mathrm{~K}$; (iii) a blue HB (BHB) morphology, peaked at $20000 \mathrm{~K}$ and with a tail of hot sdB stars, up to a temperature of $40000 \mathrm{~K}$ (see Buzzoni 1989, , for further details).

Interestingly enough, the comparison with the observations shows that "UV-upturn" ellipticals cannot be compatible with any BHB morphology alone. Too many hot stars would make the $1550 \AA$ A galaxy luminosity exceedingly bright compared to the energy bulk released at the $V$ band. On the other hand, neither an IHB is viable, given the catastrophic impact of its A-star component on the integrated $H \beta$ index of the population (e.g. Rose \& Deng 1999). Definitely, an optimum match to these systems requires a mixed contribution, where the bulk of the galaxy luminosity (over $80 \%$ ) is provided by "standard" (metal-rich) stellar populations with a red $\mathrm{HB}$, upon which a $20 \%$, or less, in terms of total optical contribution, BHB component is superposed. Figure 10 provides an illustrative picture of this case, showing the synthetic c-m diagram and the integrated SED for the composite stellar population corresponding to the big triangle in Fig. 9.

So, in full agreement with Brown's (2004) conclusions, a somewhat dichotomic scenario seems to emerge for giant ellipticals, where the occurrence of the "UVupturn" phenomenon is intimately related to the appearence, among the galaxy HB population, of a hot and very low-mass stellar component $\left(\mathrm{M}_{*} \ll 0.58 \mathrm{M}_{\odot}\right.$, e.g. Sweigart \& Gross 1976), that at some stage begins to join the standard red-clump stars. It could be useful to note, in this regard, that two metallicity ranges seem to favor the formation of EHB stars, through an optimum combination of MSTO stellar masses and subsequent mass-loss via stellar winds along the RGB. As displayed in Fig. 11, $[\mathrm{Fe} / \mathrm{H}]$ values of either $\simeq-0.7$ dex or $\gtrsim+0.5$ provide in principle the needed low mass at the onset of HB evolution in a standard Reimers mass-loss framework with $\eta \gtrsim 0.5$. 


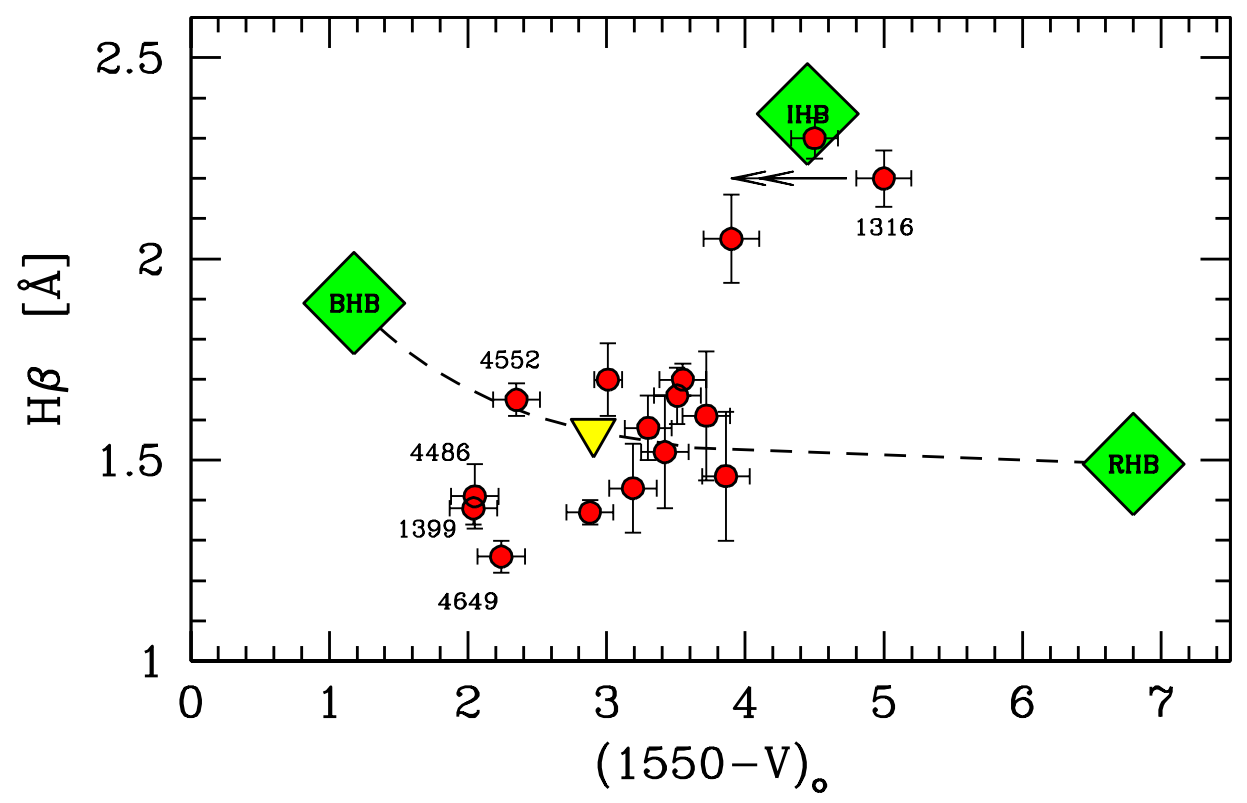

Fig. 9. - Lick H $\beta$ index vs. (reddening corrected) UV color $(1550-V)_{o}$, for the galaxy sample in Table 2 . Three reference SSP models are superposed, after Buzzoni (1989), exploring different HB morphologies (namely a red, RHB, an intermediate, IHB, and a blue, BHB, temperature distributions, as explained in the text), assuming a $15 \mathrm{Gyr}$, slightly metal-rich $([\mathrm{Fe} / \mathrm{H}]=+0.2)$ and with a fixed Reimers mass-loss parameter $(\eta=0.3)$ stellar population. Note that the bulk of "UV-upturn" ellipticals need a mixture of blue and red HB stars, roughly in a proportion of $[\mathrm{N}(\mathrm{RHB}): \mathrm{N}(\mathrm{BHB})]=[80: 20]$, as marked by the big triangle in the plot. For this composite stellar population, Fig. 10 reports the resulting synthetic c-m diagram and the integrated SED.
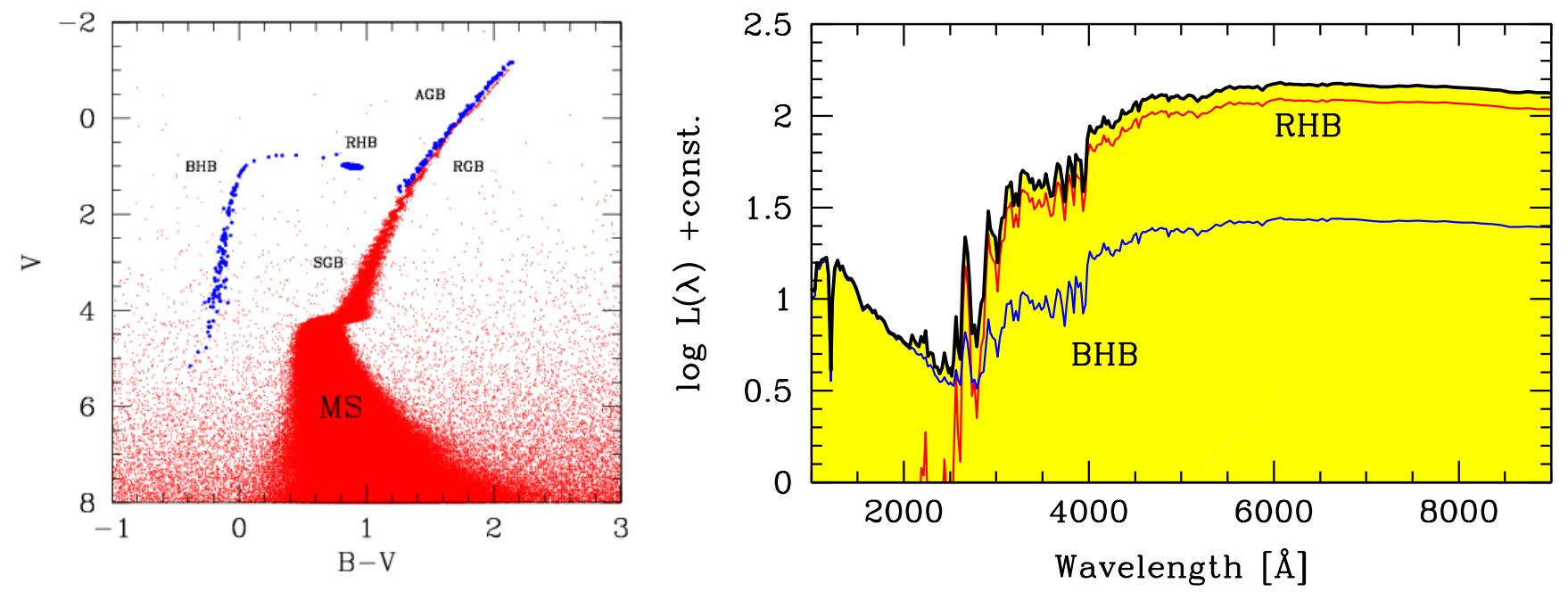

FIG. 10.- Left panel - Synthetic c-m diagram for the reference composite stellar population matching "UV-upturn" ellipticals, as discussed in Fig. 9 (the corresponding big triangle in the plot), after Buzzoni (1989). The resulting mix assumes a bimodal HB morphology, with a prevailing bulk of red HB (RHB) stars and a hot tail (BHB), extending up to $T_{\text {eff }} \simeq 40000 \mathrm{~K}$. The RHB stellar component provides about $80 \%$ of the total luminosity. An age of $15 \mathrm{Gyr}$ is assumed in all cases, with a moderately metal-rich chemical composition (i.e. [Fe/H] $=+0.2$ ), a Salpeter IMF, and a fixed Reimers mass-loss parameter $\eta=0.3$. A Poissonian error is artificially added to the data to better appreciate the number density distribution of stars along the different evolutionary branches of the diagram. The integrated SED of the whole population is displayed in the right panel, disaggregating the luminosity contribution from the two star samples.

To add even further to this challenging situation, however, one also has to report that high-resolution UV spectroscopy of a sample of six UV-strong ellipticals (namely, NGC 1399, 3115, 3379, 4472, 4552, and 4649) indicates for the claimed BHB component a moderately sub-solar $\left(Z \sim 0.1 Z_{\odot}\right)$ metallicity (Brown et al. 1997). If confirmed, ${ }^{9}$ this result might lead to identify the metal-poor

9 Surface metal abundance can easily be biased by diffusion stellar component of giant ellipticals as the one responsible for the UV excess. Whatever the exact mechanism, this will cause $\mathrm{HB}$ morphology to readily assume a bimodal distribution, with a relative lack of warm stars at the intermedate $T_{\text {eff }}$ range pertinent to the A spectral type (see Fig. 10).

mechanisms in the atmosphere of sdB stars Unglaub \& Bues 2001). 


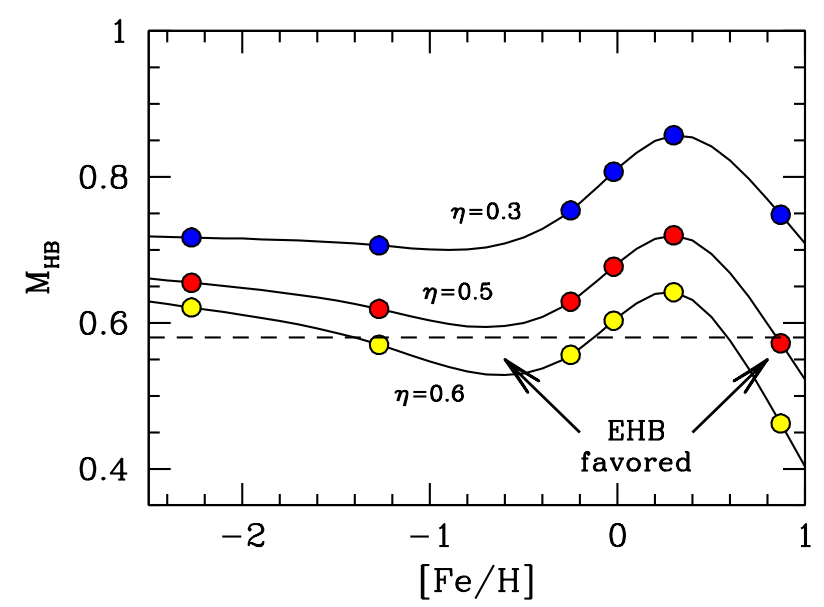

FiG. 11. - The expected representative stellar mass at the onset of the HB evolution for Buzzoni's (1989) 15 Gyr SSP models with different metallicity and mass-loss rate, according to a Reimers parameterization $(\eta)$. In combination with a moderately enhanced $\eta \simeq 0.5$ or higher) mass loss, two preferred ranges of metallicity (namely $[\mathrm{Fe} / \mathrm{H}] \simeq-0.7$ dex and $\gtrsim+0.5$ ) may more easily favor the presence of low HB masses $\left(M_{H B} \lesssim 0.58 \mathrm{M}_{\odot}\right.$, see dashed line in the plot) and the corresponding appearence of a hot-temperature tail in the HB morphology.

\section{SUMMARY AND CONCLUSIONS}

In this paper we have carried out a synoptic analysis of the different observational features that have to do with the UV luminosity excess in elliptical galaxies. As far as the canonical picture is assumed, with old stellar populations dominating early-type galaxy luminosity, the appearence of the "UV upturn" should readily call for a profound change in the c-m diagram of galaxy stellar populations, not only involving the hot stellar component of the galaxy but also reverberating on red-giant evolution at the low-temperature regime. As we mainly deal with distant, unresolved stellar populations, our analysis has to rely on a combined approach, matching infrared and ultraviolet diagnostic tools in order to probe the main features of the stellar c-m diagram, starting with integrated galaxy photometry.

Theory of surface-brightness fluctuations provides, in this sense, a natural and quite powerful way to go deep inside the problem and, as far as the infrared wavelength interval is considered, we have demonstrated theoretically that a straight and very clean relationship is in place between a macroscopic measure, such as the galaxy fluctuation magnitude, and the corresponding individual magnitude of the brightest stars in turn at the tip of the red-giant (AGB+RGB) phases (Fig. 2).

Played in the $K$ band, this correlation leads, from a measurement of $\bar{K}$, to a value for $K_{\text {tip }}$ :

$$
K_{\mathrm{tip}}=0.75 \bar{K}-3.1 \text {, }
$$

with a $\pm 0.2 \mathrm{mag}$ internal uncertainty. As we showed in Sec. 2, our SSP theoretical predictions find full support from the observations, and a direct check on the MC star clusters confirms the $\bar{K}$ vs. $K_{\text {tip }}$ relationship to be a much more general and deeply intrinsic property of stellar populations, virtually independent from any assumption about age, metallicity, IMF, and mass loss parameters. Given its nature, this relationship cannot, by itself, help disentangle the problem of age/metallicity

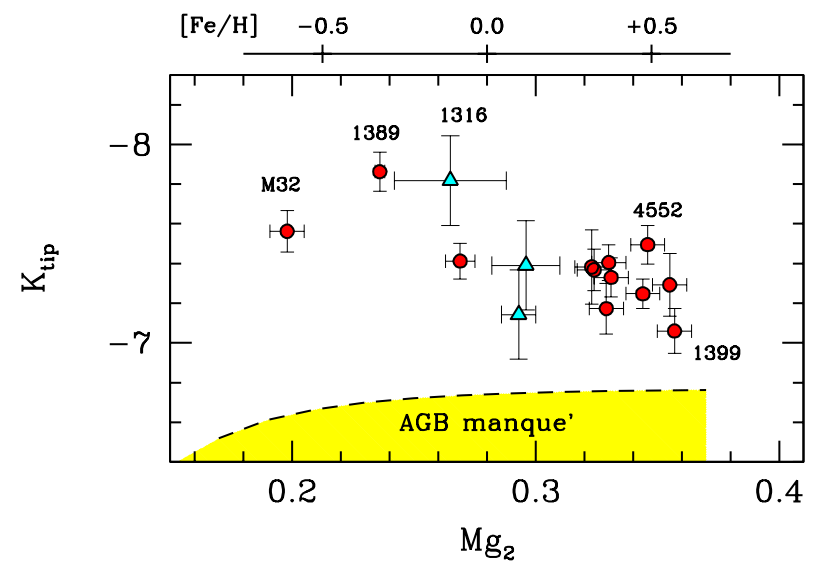

FIG. 12.- $K_{\text {tip }}$ vs. Lick $\mathrm{Mg}_{2}$ index, for the galaxy sample in Table 2 The Lick index is assumed to trace galaxy metallicity according to the Buzzoni et al. (1992) calibration, as reported on the top axis of the plot. The observed decrease in $K_{\text {tip }}$ with increasing $[\mathrm{Fe} / \mathrm{H}]$ can be mostly explained if more metal-rich galaxies are also older, as in a standard monolythic scenario for galaxy formation. The dashed line on the plot marks the minimum luminosity required for stars to experience the thermal pulsing phase along their AGB evolution, and thus end their evolution as PNe.

degeneracy; however, quite fruitfully, it provides us with a very direct probe of AGB properties, in a number of relevant details that directly deal with the mass-loss impact and the mass of dying stars (Fig. 4).

Our effort toward exploring the infrared side of galaxy SEDs has a twofold aim since, as a consequence of the basic principle of energy conservation, any gram of stellar fuel spent to feed ultraviolet luminosity cannot (and will not) be spent at longer wavelengths. This has led to the key issue of this paper, summarized in Fig. 6, that the strengthening of the $U V$ rising branch is always seen to correspond to a weakening in the AGB luminosity extension, as traced by galaxy $K$ fluctuation magnitude.

This "shortening" in AGB deployment is mainly recognized among giant ellipticals $(\bar{K}$ becomes fainter with increasing galaxy velocity dispersion, $\sigma_{v}$, see Fig. 7), and could mainly be ascribed to an age effect, as the AGB tip naturally fades in luminosity with increasing age of the system (Fig. 3), and high-mass galaxies are recognized to be older than systems of lower mass (e.g. Burstein et al. 1988; Bressan et al. 1996; Liu et al. 2002; Jensen et al. 2003; González-Lópezlira et al. 2005; Renzini 2006). However, the relationship in place likely calls for a more elaborated physical scenario, once the full range of observing evidences is added to our analysis.

(a) Besides being old, "UV upturn" galaxies are also metal rich (i.e., stronger in $\mathrm{Mg}_{2}$ Lick index). Disregarding any change in mass-loss rate, stellar tracks predict slightly more massive stars to evolve off the MS at a fixed age, with increasing metallicity (e.g., Bressan et al. 1994; Cantiello et al. 2003). This leads to correspondingly more massive AGB stars and a brighter AGB luminosity. Facing the observed trend in galaxy distribution, as summarized in Fig. 12, metallicity effects evidently enter by mitigating the dimming action of age on $K_{\text {tip }}$ with increasing galaxy mass. In any case, the interplay between age and metal abundance actually makes the derived range for the Reimers mass-loss parameter (i.e. $\Delta \eta \simeq 0.4$, as discussed in Sec. 3) a safe upper limit. In 


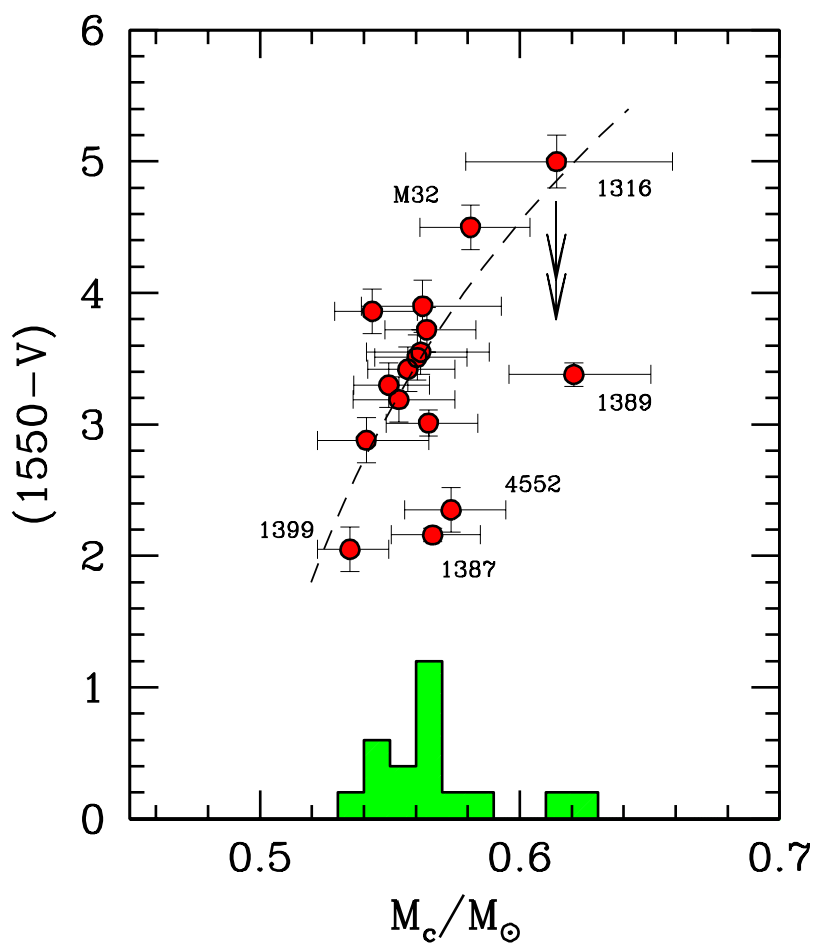

FIG. 13.- Observed ultraviolet color $(1550-V)$ vs. core mass of stars at the AGB luminosity tip, as inferred from eq. (8), for the elliptical galaxy sample of Table 2 . The $\mathrm{M}_{c}$ distribution is summarized in the lower histogram, and is the maximum actual mass allowed to luminous stars in the galaxies. One sees that mass of dying stars tends to decrease with increasing UV-upturn strength, being in general $\lesssim 0.57 \mathrm{M}_{\odot}$ among giant ellipticals. The "outlier" objects of Fig. 6 are identified again here, with galaxies labelled according to their NGC number. The arrow for NGC 1316 accounts for the claimed strong internal reddening for this galaxy. Displayed uncertainties for the derived values of $\mathrm{M}_{c}$ take in the full error budgeteach component of $\sigma\left(M_{c}\right)$ being added in quadrature-, including the contribution of $\bar{K}$ observations, $K$-band bolometric correction $(\sigma= \pm 0.2 \mathrm{mag})$, and the $K_{\text {tip }}$ vs. $\bar{K}$ calibration $(\sigma= \pm 0.2 \mathrm{mag})$. See text for a discussion.

fact, it suggests that metal abundance does not modulate by orders of magnitude mass-loss efficiency via stellar winds. ${ }^{10}$

(b) A match of galaxy $\bar{K}$ data with the calibration of Fig. 5 confirms that, all the way, brightest stars in ellipticals are genuine AGB members, reaching the thermalpulsing phase (see also Fig. 12), and with the AGB tip exceeding the RGB tip by some 0.5-1.5 mag. In the temperature range of $\mathrm{M}$ giant stars, a major fraction of bolometric luminosity is emitted through the $K$ band, and bolometric correction is a nearly constant quantity that we can estimate from $(\mathrm{Bol}-K)=+2.75 \pm 0.2 \mathrm{mag}$ (Johnson 1966); we can therefore straightforwardly translate the

\footnotetext{
${ }^{10}$ Observational evidence about the link between metallicity and mass-loss in the Milky Way and the Magellanic Clouds is contradictory. For example, Groenewegen et al. (1995) find indications that mass-loss rate (not necessarily mass-loss efficiency) in single AGB stars is linearly proportional to $Z$. Conversely, also from data of single stars, van Loon (2000) argues that $\dot{M}$ is metallicityindependent. From a theoretical point of view, Cantiello et al. (2003) models suggest that, if mass-loss is really proportional to metallicity, its effect to dim near-IR effective luminosities on average almost exactly offsets the brightening effect of metallicity itself.
}

galaxy fluctuation magnitude into an estimate of the bolometric tip luminosity, $L_{*}^{\mathrm{tip}}$, and therefrom of the corresponding stellar core mass. ${ }^{11}$ From our previous calibration (eq. 6), we can write then

$$
\log L_{*}^{\mathrm{tip}}=-0.4[(0.75 \bar{K}-3.1)+2.75-4.72]
$$

(where the Sun has magnitude $\mathrm{M}_{\mathrm{Bol}, \odot}=+4.72$ ). Following Boothroyd \& Sackmann (1988), from the assumed core mass-luminosity relation for the solar metallicity range, this leads to

$$
M_{c}=\frac{L_{*}^{\mathrm{tip}}}{52000}+0.456 \quad\left[M_{\odot}\right] .
$$

Figure 13 reports the inferred core-mass distribution, at the PN onset, for our galaxy sample.

Note that this is the maximum actual mass allowed to luminous stars in each galaxy environment, and demonstrates that the mass of dying stars tends to decrease with increasing $U V$-upturn strength, being in general $M_{\text {dying }} \lesssim 0.57 \mathrm{M}_{\odot}$ among giant ellipticals. For this mass range, PN lifetime is the largest possible, but the timescale for the nebula to be visible is critically constrained by the transition time $\left(\tau_{\mathrm{tt}}\right)$ needed by the post-AGB stellar core to be hot enough to "fire up" the ejected envelope and become a hard UV emitter (Stanghellini \& Renzini 2000; Marigo et al. 2004). The evident drop of $\alpha$ among strong UV-upturn galaxies (Fig. 8) might be a direct consequence, therefore, of an increasing blocking effect of $\tau_{\mathrm{tt}}$ along the inferred $M_{c}$ range (i.e., the stellar core takes longer to heat-up than the shell to evaporate; Buzzoni et al. 2006), to which one has to further add a size cut in the overall PN population, as a result of the EHB progenitors $\left(\mathrm{M}_{*} \lesssim 0.52 \mathrm{M}_{\odot}\right)$ evolving as AGB-manqué stars and therefore skipping the nebula event.

(c) We remarked, in Sec. 3, the importance of the integrated $H \beta$ index as a fairly selective tracer of the warm $\left(\mathrm{T}_{\text {eff }} \simeq 8000-10000 \mathrm{~K}\right)$ stellar component in the galaxy stellar population. A proper assessment of the photometric contribution from this range of temperatures is of paramount importance in the framework of early-type galaxy evolution, in order to single out any signature of recent (i.e., in the last few Gyr or so) star formation or, conversely, of intervening evolution of the HB morphology among old SSPs, as in a more standard canonical scenario.

As far as UV-upturn galaxies are concerned, the study of $H \beta$ distribution clearly points to a substantial lack of A-type stars in the galaxy mix (see Fig. 9). While, on one hand, this definitely secures the "quiescent" nature of these galaxies, it also poses, on the other hand, a stringent constraint on HB morphology in their old-age context. In fact, a bimodal temperature distribution is required for the HB to assure, at a time, both an enhanced UV emission and a conveniently low $H \beta$ feature. Thus, in these systems the expected prevailing bulk of red HB stars should be accompanied, at some point, by

\footnotetext{
11 A somewhat linear relationship between stellar luminosity and core mass is a general consequence of any evolutionary stage characterized by a (multi) shell-burning regime in the presence of a relatively thin external envelope (Paczyński 1970). This is actually the case of both pre-He flash evolution along the RGB and the thermal pulsing phase along the AGB (see Iben \& Renzini 1983; Boothrovd \& Sackmann 1988, , for a more general discussion).
} 
a residual population of blue (metal-poor?) HB objects (coincident with EHB stars in the current empirical classification scheme), peaked at about $20000-40000 \mathrm{~K}$, in a proportion of, roughly, $N_{\mathrm{RHB}}: N_{\mathrm{BHB}} \simeq[80: 20]$.

On the other hand, to complete the picture, one cannot neglect the masking effects of age distribution, facing a recognized evidence for low-mass ellipticals to display a more silent but also more continuous star formation along their entire galaxy life, that naturally feeds the A-star contribution, thanks to the bluer MS turnoff point (MSTO) exhibited by SSPs in the $1-3 \mathrm{Gyr}$ age range, and leads to a younger "average" age, compared to high-mass systems. This is what we observe, for instance, among the resolved stellar populations of the Local Group dwarf spheroidals (Mateo 1998) (see, in this regard, the illustrative location of M32 in Fig. 9, and also consider the discussion by Schiavon et al. 2004).

(d) Along with our discussion of the $\bar{K}$ vs. (1550$V)$ relation, we noticed, in Fig. 6, the presence of a few outliers about 0.7 mag brighter at infrared magnitudes, or alternatively $\sim 1.5 \mathrm{mag}$ "bluer" in the $(1550-V)$ color, than the main galaxy population. In order to further investigate this issue, we tracked the relevant objects also in other figures, whenever possible.

If galaxy mass (alias $\log \sigma_{v}$ ) is considered as the leading physical parameter to compare outlier location with respect to the bulk of the galaxy distribution (see, for instance Fig. 7), one must conclude that both NGC 4552 and NGC 1389 seem to have a brighter AGB tip rather than a bluer $(1550-V)$ color. With regard to NGC 1389 , Liu et al. (2002) find that the $\overline{K_{s}}$ SBFs of NGC 1389 are also too bright compared to its $(V-I)$ color, a fact that would be consistent with either a higher than average metallicity given the age of its most recent burst of

\footnotetext{
12 Jensen et al. (1996) attribute the difference to the higher S/N ratio of their data; the $\mathrm{S} / \mathrm{N}$ ratio of the Liu et al. (2002) images is similar to that of Jensen et al.'s.

13 Note that NGC 1387 complies with the $\bar{K}_{s}$ vs. $(V-I)$ correlation determined by Liu et al. (2002).

14 The other galaxies in our sample are all genuine ellipticals
}

star formation or a longer lifetime of its TP-AGB stars (Mouhcine et al. 2005). In the case of NGC 4552, however, Jensen et al. (1996) contribute an interesting piece of information. These authors measure near-IR SBFs for several galaxies in Virgo, and their results are systematically fainter than those obtained by Pahre \& Mould (1994). Unfortunately, the two groups use slightly different filters (Jensen et al. employ $K^{\prime}$, vs. $K_{s}$ of Pahre $\&$ Mould), but the discrepancy is larger than can be ascribed to the effect of the filters. ${ }^{12}$ In particular, Jensen et al. find $\overline{K^{\prime}}=-5.51$ for NGC 4552 ; assuming $\overline{K^{\prime}} \equiv \overline{K_{s}}$, NGC 4552 would no longer be deviant in the $\overline{K_{s}}$ vs. $(1500-V)$ plane. Concerning NGC 1387, the dearth of data for this galaxy in the literature (see Table 2) makes it hard to propose an origin for its departure from the $\overline{K_{s}}$ vs. $(1500-V)$ sequence. ${ }^{13}$ On the other hand, NGC 1387 and NGC 1389 are lenticular galaxies, like the merger remnant NGC 1316, but so are NGC 3384 and NGC 4406, both of which do not deviate from the correlation. ${ }^{14}$ At any rate, NGC 1387 and NGC 1389 constitute privileged candidates for any future "in-depth" investigation.

We would like to thank Gustavo Bruzual for providing us with his latest SSP models, in advance of publication, and Livia Origlia, for useful discussions. The anonymous referee is also acknowledged for his/her competent suggestions, that greatly helped refine the main focus of the paper. Partial financial support is acknowledged from the Italian MIUR, under grant INAF-PRIN05 1.06.08.03, and Mexican CONACyT, under grant no. 48589-F, and DGAPA, under grant IN111007.

(de Vaucouleurs et al. 1991), except for NGC 224 (alias M 31), which is so close, however, that bulge SBFs can be measured without any significant contamination from the disk.

\section{REFERENCES}

Bailyn, C. D. 1995, ARA\&A, 33, 133

Blakeslee, J. P., Vazdekis, A., \& Ajhar, E. A. 2001, MNRAS, 320, 193

Boothroyd, A. I., \& Sackmann, I.-J. 1988, ApJ, 328, 641

Bressan, A., Chiosi, C., \& Fagotto, F. 1994, ApJS, 94, 63

Bressan, A., Chiosi, C., \& Tantalo, R. 1996, A\&A, 311, 425

Brodie, J. P., \& Huchra, J. P. 1990, ApJ, 362, 503

Brown, T. M. 2004, Astrophys. Space Sci., 291, 215

Brown, T. M., Ferguson, H. C., Davidsen, A. F., Dorman, B. 1997 482, 685

Brown, T. M., Ferguson, H. C., Stanford, S. A., Deharveng, J.-M. 1998, AJ, 504, 113

Brown, T. M., Bowers, C. W., Kimble, R. A., Sweigart, A. V., \& Ferguson, H. C. 2000, ApJ, 532, 308

Brown, T. M., Ferguson, H. C., Smith, E., Bowers, C. W., Kimble, R. A., Renzini, A., \& Rich, R. M. 2003, ApJ, 584, L69

Brown, D., Yi, S., Han, Z., \& Yoon, S.-J. 2006, Baltic Astronomy, 15,13

Bruzual, G., 2007, in proc. of the IAU Symp. No. 241 "Stellar populations as building blocks of galaxies", eds. A. Vazdekis and R. Peletier (Cambridge: Cambridge Univ. Press), p.125 (see also astro-ph/0703052)

Bruzuai, G., \& Charlot, S. 2003, MNRAS, 344, 1000

Buonanno, R., Corsi, C., Bellazzini, M., Ferraro, F. R., \& Pecci, F. F. 1997, AJ, 113, 706

Burstein, D., Bertola, F., Buson, L. M., Faber, S. M., \& Lauer, T. R. 1988, ApJ, 328, 440

Buson, L. M., Bertone, E., Buzzoni, A., \& Carraro, G. 2006, Baltic Astronomy, 15, 49
Busso, G., Moehler, S., Zoccali, M., Heber, U., \& Yi, S. K. 2005, ApJ, 633, L29

Buzzoni, A. 1989, ApJS, 71, 817

Buzzoni, A. 1993, A\&A, 275, 433

Buzzoni, A. 1995, ApJS, 98, 69

Buzzoni, A. 1998 in T. Zanzu, V. Testa \& M. Bellazzini eds., Evolving Evolution, Oss. di Cagliari, Cagliari, p. 13 (see also astro-ph/9811382)

Buzzoni, A. 2008, in D. Valls-Gabaud \& M. Chavez eds., "Resolved Stellar Populations", ASP Conf. Ser. (ASP: San Francisco), in press (see also astro-ph/0509602)

Buzzoni, A., Gariboldi, G., \& IVIantegazza, L. 1992, AJ, 103, 1814

Buzzoni, A., Mantegazza, L., \& Gariboldi, G. 1994, AJ, 107, 513

Buzzoni, A., Arnaboldi, M., \& Corradi, R. L. M. 2006, MNRAS, 368,877

Cantiello, M., Raimondo, G., Brocato, E., \& Capaccioli, M. 2003, AJ, 125, 2783

Castellani, V., \& Renzini, A. 1968, Ap\&SS, 2, 310

Castellani M., \& Tornambé A. 1991, ApJ, 381, 393

Castellani, V., Giannone, P. \& Renzini, A. 1970, Ap\&SS, 9, 418

Castellani M., Limongi M., Tornambé A. 1992, ApJ, 389, 227

Castellani, V., Iannicola, G., Bono, G., Zoccali, M., Cassisi, S., \& Buonanno, R. 2006, A\&A, 446, 569

Catelan, M., Ferraro, F. R., \& Rood, R. T. 2001, ApJ, 560, 970

Cerviño, M., \& Luridiana, V. 2006, A\&A, 451, 475

Cerviño, M., Luridiana, V., \& Castander, F. J. 2000, A\&A, 360, L5

Cerviño, M., Valls-Gabaud, D., Luridiana, V., \& Mas-Hesse, J. M. 2002, A\&A, 381, 51 
Chabrier, G. 2003, PASP, 115, 763

Charbonnel, C., Meynet, G., Maeder, A., \& Schaerer, D. 1996, A\&AS, 115, 339

Chiosi, C., Bertelli, G., Bressan, A. 1992, ARA\&A, 30,235

Code, A. D., \& Welch, G. A. 1979, ApJ, 228, 95

Cohen J. G. 1982, ApJ, 258, 143

Colina, L., Bohlin, R. C., \& Castelli, F. 1996, Instrument Science Report CAL/SCS-008 (Baltimore, STScI)

Cox, J.P. \& Giuli, R.T. 1968 Principles of Stellar Structure, Gordon \& Breach Sci. Publ. Ltd, New York.

D'Cruz, N. L., Dorman, B., Rood, R. T., \& O'Connell, R. W. 1996, ApJ, 466, 359

D'Cruz, N. L., Morgan, S. M., Böhm-Vitense, E. 2000, AJ, 120, 990

Demleitner, M., Accomazzi, A., Eichhorn, G., Grant, C. S., Kurtz, M. J., \& Murray, S. S. 2001, in Astronomical Data Analysis Software and Systems X, ASP Conf. Ser., Vol. 238, eds. F. R. Harnden, Jr., Francis A. Primini, and Harry E. Payne (San Francisco: ASP), p.321

de Vaucouleurs, G., de Vaucouleurs, A., Corwin, H.G. Jr., Buta R.J., Paturel, G., Fouque, P. 1991, Third Reference Catalog of Bright Galaxies. Springer Verlag, Heidelberg

Dorman, B., Rood, R.T., O'Connell, R.W. 1993, ApJ, 419, 596

Dorman, B., O'Connell, R. W., \& Rood, R. T. 1995, ApJ, 442, 105

Demarque, P., Mengel, J. G., \& Sweigart, A. V. 1972, ApJ, 173, L27

Elson, R. A. W., \& Fall, S. M. 1985, ApJ, 299, 211

Elson, R. A., \& Fall, S. M. 1988, AJ, 96, 1383

Ferguson, H.C., \& Davidsen, A.F. 1993, ApJ, 408, 92

Frogel, J.A., Mould, J., \& Blanco, V.M. 1990, ApJ, 352, 96

Fusi Pecci, \& F., Renzini, A. 1976, A\&A, 46, 447

González, R. A., Liu, M. C., \& Bruzual A., G. 2004, ApJ, 611, 270 .2005, ApJ, 621, 557

González-Lópezlira, R. A., Albarrán, M. Y., Mouhcine, M., Liu,M. C., Bruzual-A., G., \& de Batz, B. 2005, MNRAS, 363, 1279

Greggio, L., \& Renzini, A. 1990, ApJ, 364, 35

Groenewegen, M. A. T., Smith, C. H., Wood, P. R., Omont, A., \& Fujiyoshi, T. 1995, ApJ, 449, L119

Hui X., Ford H.C., Ciardullo R., Jacoby G.H. 1993, ApJ, 414, 463

Iben, I. J., \& Rood, R. T. 1970, ApJ, 161, 587

Iben, I., Jr., \& Renzini, A. 1983, ARA\&A, 21, 271

Jensen, J. B., Luppino, G. A., \& Tonry, J. L. 1996, ApJ, 468, 519

Jensen, J. B., Tonry, J. L., \& Luppino, G. A. 1998, ApJ, 505, 111

Jensen, J. B., Tonry, J. L., Barris, B. J., Thompson, R. I., Liu, M. C., Rieke, M. J., Ajhar, E. A., \& Blakeslee, J. P. 2003, ApJ, 583,712

Johnson, H. L. 1966, ARA\&A, 4, 193

Kaluzny, J., \& Udalski, A. 1992, Acta Astronomica, 42, 29

Kobayashi, C., \& Arimoto, N. 1999, ApJ, 527, 573

Kuntschner, H., Lucey, J. R., Smith, R. J., Hudson, M. J., \& Davies, R. L. 2001, MNRAS, 323, 615

Larson, R.B. 1974, MNRAS, 166, 585

Lee, M. G., Freedman, W. L., \& Madore, B. F. 1993, ApJ, 417, 553

Lee, Y.-W., Ree, C. H., Rich, R.M., et al. 2005, ApJ, 619, L103

Liu, M. C., Graham, J. R., \& Charlot, S. 2002, ApJ, 564, 216

Maraston, C.. Greggio, L., \& Thomas, D. 2001, Ap\&SS, 276, 893

Marigo, P., Girardi, L., Weiss, A., Groenewegen, M. A. T., \& Chiosi, C. 2004, A\&A, 423, 995
Marigo, P., \& Girardi, L. 2007, A\&A, 469, 239

Mateo, M. L. 1998, ARA\&A, 36, 435

Mouhcine, M., González, R. A., \& Liu, M. C. 2005, MNRAS, 362, 1208

O'Connell, R. W. 1980, ApJ, 236, 430

O'Connell, R. W. 1999, ARÀ\&A, 37, 603

Paczyński, B. 1970, Acta Astronomica, 20, 47

Pahre, M. A., de Carvalho, R. R., \& Djorgovski, S. G. 1998, AJ, 116,1606

Pahre, M. A., \& Mould, J. R. 1994, ApJ, 433, 567

Park, J.-H., \& Lee, Y.-W. 1997, ApJ, 476, 28

Paturel, G., Petit, C., Prugniel, P., Theureau, G., Rousseau, J., Brouty, M., Dubois, P., \& Cambrésy, L. 2003, A\&A, 412, 45

Peimbert M. 1990, Rev. Mex. Astron. Astrof., 20, 119

Persson, S. E., Murphy, D. C., Krzeminski, W., Roth, M., \& Rieke,

M. J. 1998, AJ, 116, 2475

Piersanti, L., Tornambé, A., \& Castellani, V. 2004, MNRAS, 353, 243

Raimondo, G., Brocato, E., Cantiello, M., \& Capaccioli, M. 2005, AJ, 130, 2625

Recio-Blanco, A., Aparicio, A., Piotto, G., de Angeli, F., \& Djorgovski, S. G. 2006, A\&A, 452, 875

Ree, C. H., Lee, Y.-W., Sohn, Y.-J., Yi, S. K., Yoon, S.-J., \& the GALEX Science Team 2006, Baltic Astronomy, 15, 5

Reimers, D. 1975, Mem. Soc. Roy. Sci. Liège, 6th Ser., 8, 87

Renzini, A. 1977, in Advanced Stages in Stellar Evolution I., eds. Iben Jr., A. Renzini, D.N. Schramm (Heidelberg: Springer Verlag).

Renzini, A. 2006, ARA\&A, 44, 141

Renzini, A., \& Buzzoni, A. 1986, in Spectral Evolution of Galaxies, eds. C. Chiosi \& A. Renzini (Dordrecht: Reidel), p. 195

Renzini, A., \& Fusi Pecci, F. 1988, ARA\&A, 1988, 26, 199

Rich, R. M., et al. 1997, ApJ, 484, L25

Rose, J.A., \& Deng, S. 1999, AJ, 117, 2213

Schönberner, D. 1983, ApJ, 272, 708

Schiavon, R.P., Caldwell, N., \& Rose, J.A. 2004, AJ, 127,1513

Searle, L., Wilkinson, A., \& Bagnuolo, W. G. 1980, ApJ, 239, 803

Seidel, E., Da Costa, G. S., \& Demarque, P. 1987, ApJ, 313, 192

Skrutskie, M. F. et al. 1997, ASSL Vol. 210: The Impact of Large Scale Near-IR Sky Surveys, 25

Stanghellini, L., \& Renzini, A. 2000, ApJ, 542, 308

Sweigart, A. V. 1997, ApJ, 474, L23

Sweigart, A. V., \& Gross, P. G. 1976, ApJS, 32, 367

Sweigart, A. V. Brown, T. M., Lanz, T., Landsman, W. B., Hubeny, I. 2002 in Omega Centauri, a unique window into astrophysics. ASP Conf. Ser., Vol. 265. Eds. F. van Leeuwen, J. D. Hughes, \& G. Piotto (San Francisco: ASP) p.261

Tantalo, R., Chiosi, C., Bressan, A., \& Fagotto, F. 1996, A\&A, 311,361

Tonry, J. L. 1991, ApJ, 373, L1

Tonry, J., \& Schneider, D. P. 1988, AJ, 96, 807

Unglaub, K., Bues, I. 2001, A\&A, 374, 570

Van den Bergh, S. 1962, AJ, 67, 486

van Loon, J. T. 2000, A\&A, 354, 125

Worthey, G. 1993, ApJ, 409, 530

Worthey, G. 1994, ApJS, 95, 107

Yi, S. K., \& Yoon, S.-J. 2004, Ap\&SS, 291, 205

Yi S., Demarque P., Oemler A. J. 1998, ApJ, 492, 480 